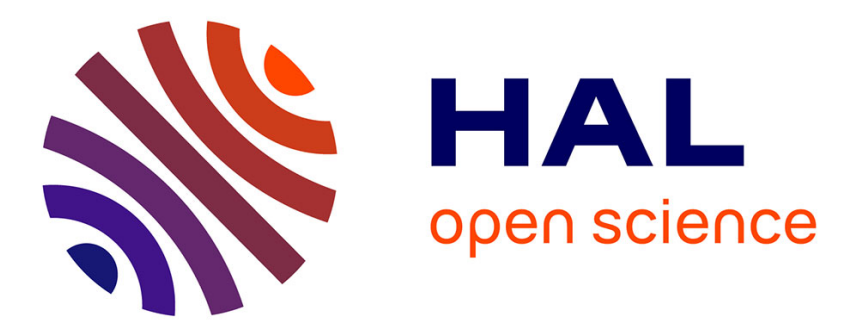

\title{
Compilation and critical analysis of thermodynamic data for ternary alloy systems
}

I. Ansara, J.P. Bros, P. Spencer

\section{To cite this version:}

I. Ansara, J.P. Bros, P. Spencer. Compilation and critical analysis of thermodynamic data for ternary alloy systems. [Research Report] LTPCM. 1974, 37 p. hal-01364060

\section{HAL Id: hal-01364060 \\ https://hal.science/hal-01364060}

Submitted on 12 Sep 2016

HAL is a multi-disciplinary open access archive for the deposit and dissemination of scientific research documents, whether they are published or not. The documents may come from teaching and research institutions in France or abroad, or from public or private research centers.
L'archive ouverte pluridisciplinaire HAL, est destinée au dépôt et à la diffusion de documents scientifiques de niveau recherche, publiés ou non, émanant des établissements d'enseignement et de recherche français ou étrangers, des laboratoires publics ou privés. 


\section{$R P 134(4)$}

INSTITUT NATIONAL POLYTECHNIQUE DE GRENOBLE

\section{LABORATOIRE DE THERMODYNAMIQUE}

\section{ET PHYSICO-CHIMIE METALLURGIQUES}

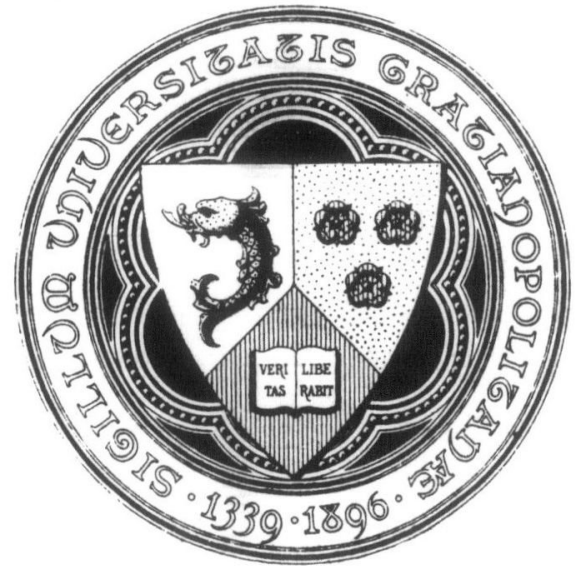

COMPILATION AND CRITICAL ANALYSIS OF THERMODYNAMIC DATA FOR TERNARY ALLOY SYSTEMS

by

I. ANSARA, J.P. BROS and P. SPENCER

(presented at the IVth International CODATA conference

TSACHKADZOR, Armenia Republic, U.S.S.R., 24-27 June 1974)

REFERENCE. RAPPORT LTPCM-1974-TM-04

ENSEEG-B.P.44-38401 ST . MARTIN D'HERES-FRANCE 
COMPILATION AND CRITICAL ANALYSIS OF THERMODYNAMIC DATA FOR TERNARY ALLOY SYSTEMS

by

I. ANSARA, J.P. BROS* and P. SPENCER**

* U.E.R. Chimie - Université de Provence - Place V. Hugo, 13003 Marseille, France.

** National Physical Laboratory, Teddington, Middlesex,U.K. 
The thermodynamic approach in resolving industrial problems concerned with high temperature has led, in the two last decades, to a notable development of both theoretical and experimental studies on multicomponent systems. Phase diagram determinations of metallic systems are still carried out very extensively because of their importance in the field of material science, mainly in steelmaking, non-ferrous metallurgy, crystal growth, electroslagrefining, nuclear materials, etc...

These phase diagrams, usually determined by classical methods such as thermal analysis, X-ray cristallography, equilibrium techniques, are established in relatively small composition and temperature ranges. It is obvious that a complete study of such multicomponent systems, useful for the scientist, is tedious and time-consuming. These experimental results have been reviewed in the compilations published by Janecke ${ }^{(1)}, \operatorname{Rudy}^{(2)}$, Agaev ${ }^{(3)}$, Guertler ${ }^{(4)}$, and it is desirable that such reviews continue.

Phase diaqrams calculated from the thermodynamic properties of mixing are less numerous because of the lack of experimental determinations of such properties for ternary systems, although there has been extensive work on the prediction of phase boundaries obtained from the limiting binary systems ${ }^{(5-23)}$.

These two approaches, which can be reconciled through thermodynamic principles, have most of the time been carried out independently neglecting the thermodynamic data relative to the multicomponent solution phase. And yet, from the literature, it can be seen that measurements of the thermodynamic properties of mixing mainly in homogeneous metallic multicomponent phases has been increasing in recent years, the results of which permit a more complete description of a system.

At the second and third CODATA Conferences $(24,25)$ an international cooperative research program dealing with the compilation, critical evaluation and computer storage of the thermodynamic properties of the elements, 
inorganic compounds and solution phases was described. In relation to this program, a projectfor the compilation, evaluation and tabulation of the thermodynamic properties of ternary metallic systems is described in this paper. This project excludes, for the time being non-metallic systems, salt and oxide systems .

The aim of this project is to achieve a uniform presentation of the thermodynamic data for alloys and therefore:

- obtain missing binary data by extrapolation of the ternary values

- compute ternary and multicomponent phase diagrams

- correlate thermodynamic data with experimental phase

diagrams

- investigate the influence of a third element on a binary system from the view point of bond mechanism.

At the moment, experimental data for multicomponent systems are presented in variety of ways, for example :

- tables of $\Delta \bar{G}_{i}=f\left(T, x_{i}, x_{j} / x_{k}, \ldots\right)$

- tables of $\Delta \bar{G}_{i}=f\left(T, x_{i}, x_{j}, x_{k} \ldots\right)$

- analytical expressions for $\Delta \bar{G}_{i}, \Delta H$, etc... derived from various physico-chemical models or more simply, from surface fitting

- graphical representation.

Moreover, in inteqrating the Gibbs-Duhem equation for the partial properties of solution, a number of methods are available ${ }^{(26-30)}$, all of which usually require limiting binary data. Precise details of the binary data and procedures actually used are rarely qiven in published papers. Similar remarks also apply in respect of the interpolation and differentiation of experimental inteqral quantities.

A feature which is rarely achieved is the consistency between the thermodynamic properties of solution and ternary phase equilibria. This is 
due, not only to the lack of complete information on these two properties but also to the complexity of the problem.

These considerations have led us to discuss the numerical treatment of the experimental data for the multicomponent systems. The main problem is the choice of the interpolation formula used to describe an integral thermodynamic function for such a system. In a general way, an integral excess function $\mathrm{Y}$ of solution for a ternary system can be written as follows :

$$
Y=\sum_{i} \sum_{j} \alpha Y_{i j}+x_{i} x_{j}\left(1-x_{i}-x_{j}\right) P\left(x_{i}, x_{j}\right)
$$

where $Y_{i j}$ denotes the excess function of the binary system $i-j$, in tabular or analytical form, $P\left(x_{i}, x_{j}\right)$ an equation expressing a ternary interaction contribution, $\alpha$ a weighting factor on the binary quantity which varies according to the model used $(31-41)$. Partial quantities can be derived from equation (1) and used to fit the experimental data. Normally, the values of $Y_{i j}$ are constrained by the limiting binary data, and it is the coefficients of $P\left(x_{i}, x_{j}\right)$ which are obtained by the fitting procedure.

From our experience in fitting experimental data for multicomponent solution we recommend that :

- care should be taken to use critically evaluated data for the limiting binary systems; such data are available in handbooks for example the ones published by Hultgren, and Kubaschewski $(42,43)$

- different equations, semi-empirical or preferentially derived from statistical thermodynamic be tried in onder to reduce the number of parameters in $P\left(x_{i}, x_{j}\right)$

- if suitable analytical expressions cannot be found, tables of discrete values be used together with spline function interpolation. To illustrate that, minimization of the Gibbs energy in a multicomponent system no longer requires differentiation of that function with respect to its independent variables as simplex methods $(44)$ are now readily available 
- whenever possible, phase diagrams are calculated and compared with the experimental ones

- if different sets of experimental data are available, critical evaluation be attempted.

These recommendations and procedures have long been applied for the critical evaluation of binary solution data but not always followed by experimental scientists.

Naturally, for multicomponent systems, the introduction of additional dimensions increases the difficulty and the effort required for critical evaluation, so that it is important to simplify computing procedures.

The application of the above principles is demonstrated for three ternary solutions, Cu-Fe-Ni, Ga-In-Zn, Ga-Sn-Zn in the appendix. In this treatment, the following aspects are covered.

1) Description and analysis of the experimental thermodynamic properties of the ternary system, reference being made to the experimental phase diagram.

2) Fitting of ternary data with different constraints on the binary data. Comparison between experimental and calculated values.

3) Comparison between experimental and calculated phase diagram derived from the thermodynamic data of solution.

4) Tables of the data for partial and integral heats and excess entropies of mixing.

5) Bibliography.

The above presentation is one possible procedure when sufficient information is available. Normally, however, experimental data exist only in a 
limited composition range and this procedure will not be possible in its entirety. In such cases, it is envisaged that this limited information be compiled and tabulated to await supplementary data.

The authors are aware that this form of presentation of data can be modified and comments and criticism will be greatly appreciated. Nevertheless, with more and more experimental work being undertaken in multicomponent systems, such a project, in some form, could contribute to the compilation, evaluation; tabulation of thermodynamic properties not only for alloys systems but also for oxides or salts mixtures for which much experimental data are already available.

The project described above is closely related to a nother international project CALPHAD (calculation of phase diagrams in multicomponent systems), in which American, British, French, German and Swedish scientists participate. One of their main interests is the analysis, critical evaluation and publication of consistent thermodynamic data and phase diagrams for binary systems. Such data will be used for the assessment and prediction of thermodynamic data and phase diagrams for ternary systems. A sample pamphlet for ten binary and three ternary systems is being prepared and will be diffused for criticism.

The assessment and publication of data for multicomponent systems is an ambitious task. However, it is felt that with the increasing use of computer techniques, a relatively modest effort in this field would provide a method of making readily available to the scientific community the fruits of expensive experimental work, which is often at present difficult to exploit satisfactorily. 


\section{BIBLIOGRAPHY}

(1) E. JANECKE, "Handbuch aller Legierungen", Universitätsverlag, Heidelberg, 1949.

(2) E. RUDY, "Ternary Phase Equilibria in Transition Metal-Boron-CarbonSilicon Systems, Part V Compendium of Phase diagram Data" Tech. Rep. AFML-TR-65-2, 1969, Aerojet General Corp., Sacramento, Calif., U.S.A.

(3) N.V. AGAEV, "Phase Diagrams of Metallic Systems", 1955-1975, Inst. Inf. Sci. Techn., Moscov, URSS.

(4) W. GUERTLER, M. GUERTLER and E. ANASTASIADIAS, "A compendium of Constitutional Ternary Diagrams of Metallic Systems", WADC Tech. Rep. 58-615, 1969, U.S. Dept. Commerce, Springfield Va. 22151, U.S.A.

(5) O. KUBASCHEWSKI, G. WAINWRIGHT, F.J. KIRBY, J. Inst. Met., 1960, 89,66 .

(6) J.L. MEIJERING, Philip Res. Rep. 1951, 6, 183.

(7) L. KAUF MAN and H. BERNSTEIN, "Computer Calculation of Phase Diagrams", Academic Press, N.Y., 1970.

(8) H. GAYE, "Computer Applications of the Thermodynamic of Multicomponent Solutions", Ph.D. Thesis, Carnegie Mellon Univ., Pittsburgh, Pa., U.S.A., 1971 .

(9) J.F. COUNSELL, E.B. LEES and P.J. SPENCER, "Metallurgical Chemistry", Proc. Symp. 1971, Ed. O. Kubaschewski, HMSO, U.K., 1971, p. 451 .

(10) I. ANSARA, "Metallurgical Chemistry", Proc. Symp. 1971, Ed. O. Kubaschewski, HMSO, U.K., 1971, p. 403.

(11) J.L. MEIJERING, Acta Met., 1957, 5, 257.

(12) M. GAMBiNO, J.P. BROS and I. ANSARA, "Thermochimie", Coll. Int. CNRS, $N^{\circ} 201,1971$, Marseille, France, p. 273.

(13) M. GAMBINO, J.P. BROS, a nd I. ANSARA, Rev. Int. Iftes Temp. et Réfract., $1973,10,5$.

(14) H. GAYE and C.H.P. LUPIS, Scripta Met., 1970, 4, 685.

(15) G. KIRCHNER and B. UHRENIUS, Acta Met., 1971, 22, 523.

(16) L. KAUFMAN and H. NESOR, "Computer Analysis of Alloys Systems", Tech. Rep. AFML-TR-73-56, Manlabs Inc., Cambridge, Mass., USA

(17) I. ANSARA, P. DESRE' and E. BONNIER, C.R. Acad. Sci., 1970, 270, 1098.

(18) G.B. STRINGFELLOW and P.E. GREENE, J. Phys. Chem. Solids, 1969, 30, 1779 .

(19) G.B. STRINGFELLOW and P.E. GREENE, J. Electrochem. Sol. Sol id State Sci., 1970, 117, 1075 . 
(20) A. LAUGIER, Rev. Phys. Appl ., 1973, 8, 3, 259.

(21) A.S. JORDAN, Met. Trans., 1971, 2, 1965.

(22) Y.A. CHANG and D. NAUJOCK, Met. Trans., 1972, 3, 1693.

(23) J.F. COUNSELL, E.B. LEES and P.J. SPENCER, DCS Rep. 9, NPL, 1970, Teddington U.K.

(24) Second Int. CODATA Conference, held in St Andrews Scotland (U.K.), Sept. 1971 (no proceedings have been published).

(25) E. BONNIER, Third Int. Codata Conf., Le Creusot France, Pub. CODATA, Frankfurt/Main, BRD.

(26) L.S. DARKEN, J. Amer. Chem. Soc., 1950, 72, 2909.

(27) C. WAGNER, "Thermodynamic of Alloys", Addison Wesley Pub. Co. Inc., Mass., 1952.

(28) R. SCHUMANN, Acta Met., 1955, 3, 219.

(29) N.A. GOKCEN, J. Phys. Chem., 1960, 64, 401.

(30) N.A. GOKCEN, Aerospace Corp., Rept. N0 TR-0059 (6210-10), 2, 1971, L.A., U.S.A.

(31) F. KOHLER, Monat. Für Chemie, 1960, 91, 738.

(32) G.W. TOOP, Trans. Met. Soc. AIME, 1965, 233, 850.

(33) E. BONNIER and R. CABOZ, C.R. Acad. Sci., 1960, 250, 527.

(34) B. BRION, J.C. MATHIEU, P. HICTER and P. DESRE, J. Chim. Phys., 1969, 66,238 .

(35) B. BRION, J.C. MATHIEU, and P. DESRE , J. Chim. Phys., 1970, 67, 1745.

(36) I. ANSARA, E. BONNIER and J.C. MATHIEU, Z.fur Metallkde, 1973,64,4,258.

(37) C.H.P. LUPIS, Ph.D. Thesis, MIT, Boston 1965.

(38) C.H.P. LUPIS and J.F. ELLIOTT, Acta Met., 1967, 15, 265.

(39) K. WOHL, Trans. Amer. Inst. Chem. Eng., 1946, 42, 215.

(40) H. KEHIAIAN, Bull. Acad. Pol. Sci., 1966, 14, 153.

(41) C. COLINET, DES, Fac. Sci., Univ. Grenoble, France, 1967.

(42) R. HULTGREN, P.D. DESAI, D.T. HAWKINS, M. GLEISER and K.K. KELLEY, "Selected Values of the Thermodynamic Properties of Binary Alloys". 1973, Amer. Soc. for Metals, Metals Park, Ohio, U.S.A.

(43) O. KUBASCHEWSKI, E.LL. EVANS and C.B.J. ALCOCK, "Metallurgical Thermochemistry", Pergamon Press, 1967, U.K.

(44) J.A. NELDER and R. MEAD, Comp. J., 1965, 308. 


\section{$\begin{array}{llllllll}A & P & P & E & N & D & I & X\end{array}$}

The thermodynamic functions refer to a mole of ${ }^{A}(x){ }_{(1-x)}$

x molar fraction

$\Delta \mathrm{H}, \Delta \overline{\mathrm{H}}_{\mathrm{i}}, \Delta \overline{\mathrm{G}}_{\mathrm{i}}$ are expressed in $\mathrm{cal} . \mathrm{mol}^{-1}$ $\Delta S^{X S}, \Delta S_{i}^{X S}$ are expressed in cal. $\mathrm{mol}^{-1} \mathrm{~K}^{-1}$

Temperature in $\mathrm{K}$

$1 \mathrm{cal}=4,184 \mathrm{~J}$. 


\section{I - EXPERIMENTAL RESULTS.-}

The heats of mixing of the liquid solutions have been measured by microcalorimetry ${ }^{(1)}$ at $723 \mathrm{~K}$ for :

$$
\begin{array}{r}
0<\mathrm{x}_{\mathrm{In}}<0.8 ; \mathrm{x}_{\mathrm{Ga}} / \mathrm{x}_{\mathrm{Zn}}=1 / 3 ; 1 / 1 ; 3 / 1 \\
\text { and } 0<\mathrm{x}_{\mathrm{Zn}}<0.8 ; \mathrm{x}_{\mathrm{Ga}} / \mathrm{x}_{\mathrm{In}}=1 / 3 ; 1 / 1 ; 3 / 1
\end{array}
$$

The partial free energies have been determined ${ }^{(2)}$ by an e.m.f. method in the temperature range $693<\mathrm{T}<793 \mathrm{~K}$ for :

$$
0.1<\mathrm{x}_{\mathrm{In}}<0.9 ; \mathrm{x}_{\mathrm{Ga}} / \mathrm{x}_{\mathrm{In}}=3 / 17 ; 1 / 3 ; 3 / 1 ; 1 / 1 .
$$

\section{II-COMPARISON BETWEEN EXPERIMENTAL AND CALCULATED THERMODYNAMIC} DATA.-

\section{a) Binary solutions :}

$$
\begin{aligned}
& \operatorname{Ga-In}^{(3)}\left\{\begin{array}{l}
\Delta \mathrm{H}_{\mathrm{M}}=\mathrm{x}_{\mathrm{In}}\left(1-\mathrm{x}_{\mathrm{In}}\right)(1060) \\
\Delta S_{\mathrm{M}}^{\mathrm{xS}}=\mathrm{x}_{\mathrm{In}}\left(1-\mathrm{x}_{\mathrm{In}}\right)\left(-0.43397 \mathrm{x}_{\mathrm{In}}{ }^{2}-0.00676 \mathrm{x}_{\mathrm{In}^{-}}-0.30651\right)
\end{array}\right. \\
& G a-Z n{ }^{(1)}\left\{\begin{aligned}
\Delta \mathrm{H}_{\mathrm{M}} & =\mathrm{x}_{\mathrm{Zn}}\left(1-\mathrm{x}_{\mathrm{Zn}}\right)\left(420 \mathrm{x}_{\mathrm{Zn}}{ }^{2}+478.0 \mathrm{x}_{\mathrm{Zn}}+1413.0\right) \\
\mathrm{S}_{\mathrm{M}}^{\mathrm{XS}} & =\mathrm{x}_{\mathrm{Zn}}\left(1-\mathrm{x}_{\mathrm{Zn}}\right)\left(-9.18456 \mathrm{x}_{\mathrm{Zn}}{ }^{3}+13.08382 \mathrm{x}_{\mathrm{Zn}}{ }^{2}\right. \\
& \left.-5.35968 \mathrm{x}_{\mathrm{Zn}}+2.49791\right)
\end{aligned}\right. \\
& \operatorname{In-Zn}(4)\left\{\begin{aligned}
\Delta \mathrm{H}_{\mathrm{M}}= & \mathrm{x}_{\mathrm{Zn}}\left(1-\mathrm{x}_{\mathrm{Zn}}\right)\left(2473.5605 \mathrm{x}_{\mathrm{Zn}}{ }^{3}-2324.8502 \mathrm{x}_{\mathrm{Zn}}{ }^{2}\right. \\
& \left.+1469.9522 \mathrm{x}_{\mathrm{Zn}}+2588.1 .589\right) \\
\Delta_{\mathrm{M}}^{\mathrm{XS}}= & x_{\mathrm{Zn}}\left(1-\mathrm{x}_{\mathrm{Zn}}\right)\left(0.20367 \mathrm{x}_{\mathrm{Zn}}+1.06355\right)
\end{aligned}\right.
\end{aligned}
$$




\section{b) Ternary solutions :}

The following equation :

$$
\begin{gathered}
\Delta \mathrm{H}_{\mathrm{M}}=\left(\mathrm{x}_{\mathrm{In}}+\mathrm{x}_{\mathrm{Zn}}\right)^{2}\left(\Delta_{\mathrm{In}-\mathrm{Zn}}\right)^{\prime} \mathrm{x}_{\mathrm{In}} / \mathrm{x}_{\mathrm{Zn}}+\left(\mathrm{x}_{\mathrm{Ga}}+\mathrm{x}_{\mathrm{Zn}}\right)^{2}\left(\Delta \mathrm{H}_{\mathrm{Ga}-\mathrm{Zn} \mathrm{x}_{\mathrm{Ga}} / \mathrm{x}_{\mathrm{Zn}}}\right. \\
+\left(\mathrm{x}_{\mathrm{Ga}}+\mathrm{x}_{\mathrm{In}}\right)^{2}\left(\Delta_{\mathrm{H}}{ }_{\mathrm{Ga}-\mathrm{In}^{\prime} \mathrm{x}_{\mathrm{Ga}} / \mathrm{x}_{\mathrm{In}}}\right.
\end{gathered}
$$

fits within 25 cal the experimental data as shown in table I.

The partial free energies at $743 \mathrm{~K}$ derived from the combination of the heats and excess entropies using a similar equation are in very good agreement with the experimental values as shown in table II.

\section{Table I}

\begin{tabular}{|c|c|c|c|c|c|c|}
\hline${ }^{x} \mathrm{Ga}^{\prime}$ & \multicolumn{2}{|c|}{$1 / 3$} & \multicolumn{2}{|r|}{$1 / 1$} & \multicolumn{2}{|c|}{$3 / 1$} \\
\hline 111 & exp & exp-calc & exp & exp-calc & $\exp$ & exp-calc \\
\hline 0.1 & 570 & -11 & 580 & -15 & 380 & -3 \\
\hline 0.2 & 680 & -15 & 640 & -13 & 430 & -6 \\
\hline 0.3 & 740 & -3 & 675 & -24 & 455 & -9 \\
\hline 0.4 & 750 & -4 & 670 & -26 & 460 & -8 \\
\hline 0.5 & 725 & -5 & 635 & -29 & 450 & 3 \\
\hline 0.6 & 655 & 7 & 565 & -26 & 410 & 7 \\
\hline 0.7 & 545 & 8 & 450 & -6 & 340 & 4 \\
\hline 0.8 & 400 & 10 & 320 & 0 & 259 & 13 \\
\hline 0.9 & 220 & 11 & 175 & 3 & 130 & -4 \\
\hline
\end{tabular}

Calculated and experimental $\Delta \mathrm{H}_{\mathrm{M}}$ at $723 \mathrm{~K}(\mathrm{cal} / \mathrm{mol})$

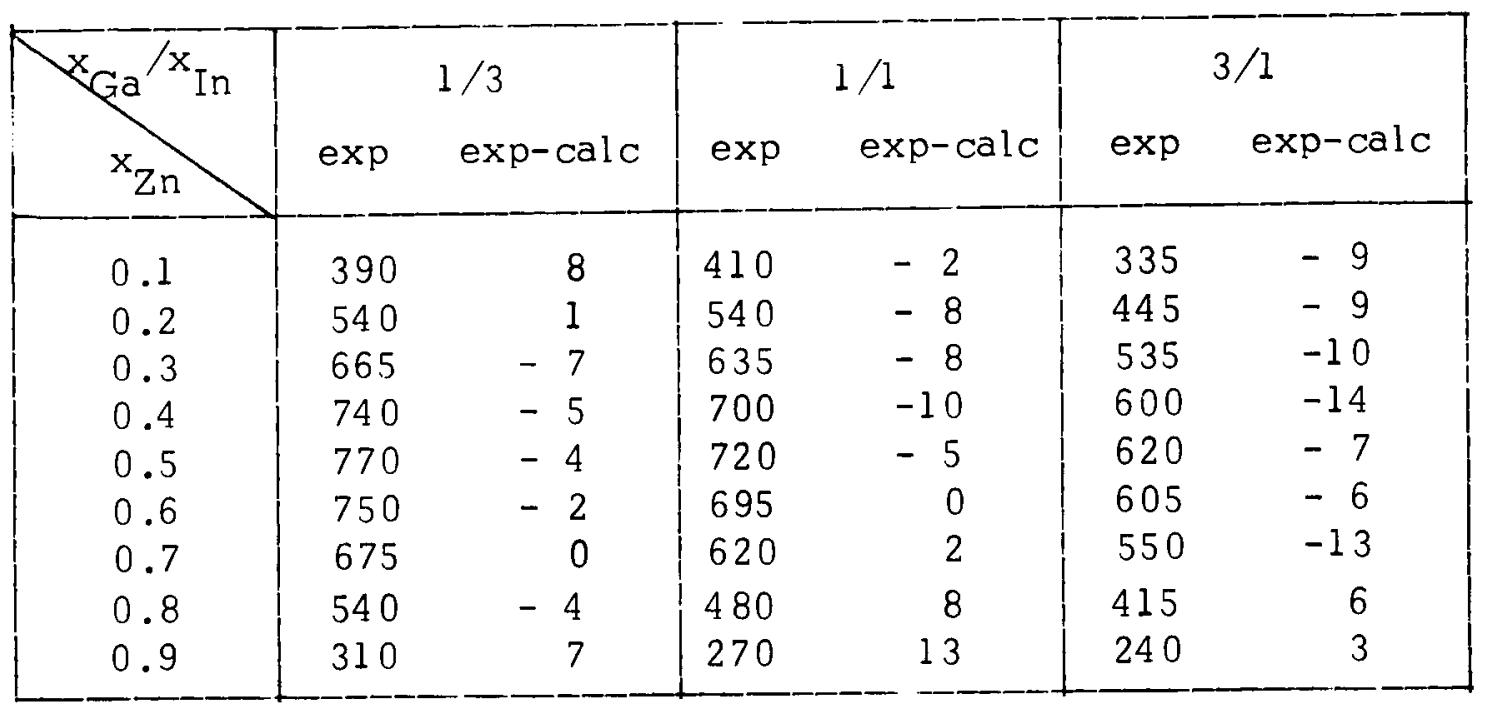


Table II

Calculated and experimental $\Delta \overline{\mathrm{G}}_{\mathrm{Zn}}$ at $743 \mathrm{~K}(\mathrm{cal} / \mathrm{mol})$

\begin{tabular}{|c|c|c|c|c|c|c|c|c|}
\hline $\mathrm{Xa}_{\mathrm{Ga}}$ & & $3 / 17$ & & $1 / 3$ & & $1 / 1$ & & $3 / 1$ \\
\hline & exp & exp-calc & exp & exp-calc & exp & exp-calc & $\exp$ & exp-calc \\
\hline 0.1 & -1995 & 125 & -2202 & 99 & -2495 & -175 & -2696 & 280 \\
\hline 0.2 & -1240 & 32 & -1187 & 262 & -1625 & -123 & -1890 & 34 \\
\hline 0.3 & -873 & -20 & - 939 & 49 & -1161 & -96 & -1320 & 87 \\
\hline 0.4 & -608 & -6 & -694 & -10 & -869 & -22 & -983 & 70 \\
\hline 0.5 & -461 & -29 & -509 & -34 & -645 & 34 & -737 & 25 \\
\hline 0.6 & -345 & -33 & -382 & -47 & -482 & 70 & -559 & -38 \\
\hline 0.7 & -272 & -42 & -296 & -52 & -345 & -62 & -419 & -82 \\
\hline 0.8 & -188 & -18 & -205 & -26 & -233 & $-\quad 37$ & -275 & -66 \\
\hline 0.9 & -115 & -8 & -112 & -1 & -126 & -10 & -137 & -23 \\
\hline
\end{tabular}

\section{III - PHASE DIAGRAM .-}

The phase diagram of the three limiting binary systems are of a eutectic type with no significant solid solubility. The properties defined above enables the liquidus on the $\mathrm{Zn}$ rich side to be calculated. These temperatures $(\mathrm{K})$ are compared in table III to those which are derived from the e.m.f. measurements ${ }^{(2)}$.

Table III

\begin{tabular}{|c|c|c|c|c|c|c|c|c|}
\hline \multirow{2}{*}{$\mathrm{Ga}_{\mathrm{Zn}}$} & \multicolumn{2}{|c|}{$3 / 17$} & \multicolumn{2}{|c|}{$1 / 3$} & \multicolumn{2}{|c|}{$1 / 1$} & \multicolumn{2}{|c|}{$3 / 1$} \\
\hline & ref(2) & exp-calc & $\operatorname{ref}(2)$ & exp-calc & $\operatorname{ref}(2)$ & exp-calc & $\operatorname{ref}(2)$ & exp-calc \\
\hline 0.1 & 471 & -5 & 443 & -2 & 405 & 2 & 380 & 6 \\
\hline 0.2 & 530 & -1 & 508 & -1 & 462 & -6 & 437 & -1 \\
\hline 0.3 & 560 & -9 & 544 & -8 & 506 & -4 & 476 & -4 \\
\hline 0.4 & 588 & -5 & 570 & -7 & 536 & -6 & 511 & -4 \\
\hline 0.5 & 603 & -7 & 590 & -7 & 560 & -12 & 539 & -4 \\
\hline 0.6 & 615 & -8 & 604 & -10 & 581 & -11 & 563 & -12 \\
\hline 0.7 & 625 & -7 & 616 & -9 & 603 & -8 & 585 & -14 \\
\hline 0.8 & 638 & -3 & 632 & -7 & 623 & -12 & 614 & -13 \\
\hline 0.9 & 654 & -7 & 655 & -4 & 650 & -7 & 647 & -10 \\
\hline
\end{tabular}

Since the differences are smaller than $15 \mathrm{~K}$, the phase diagram which is shown in the following figure has been computed (the temperaturesare in ${ }^{\circ} \mathrm{C}$ ). 


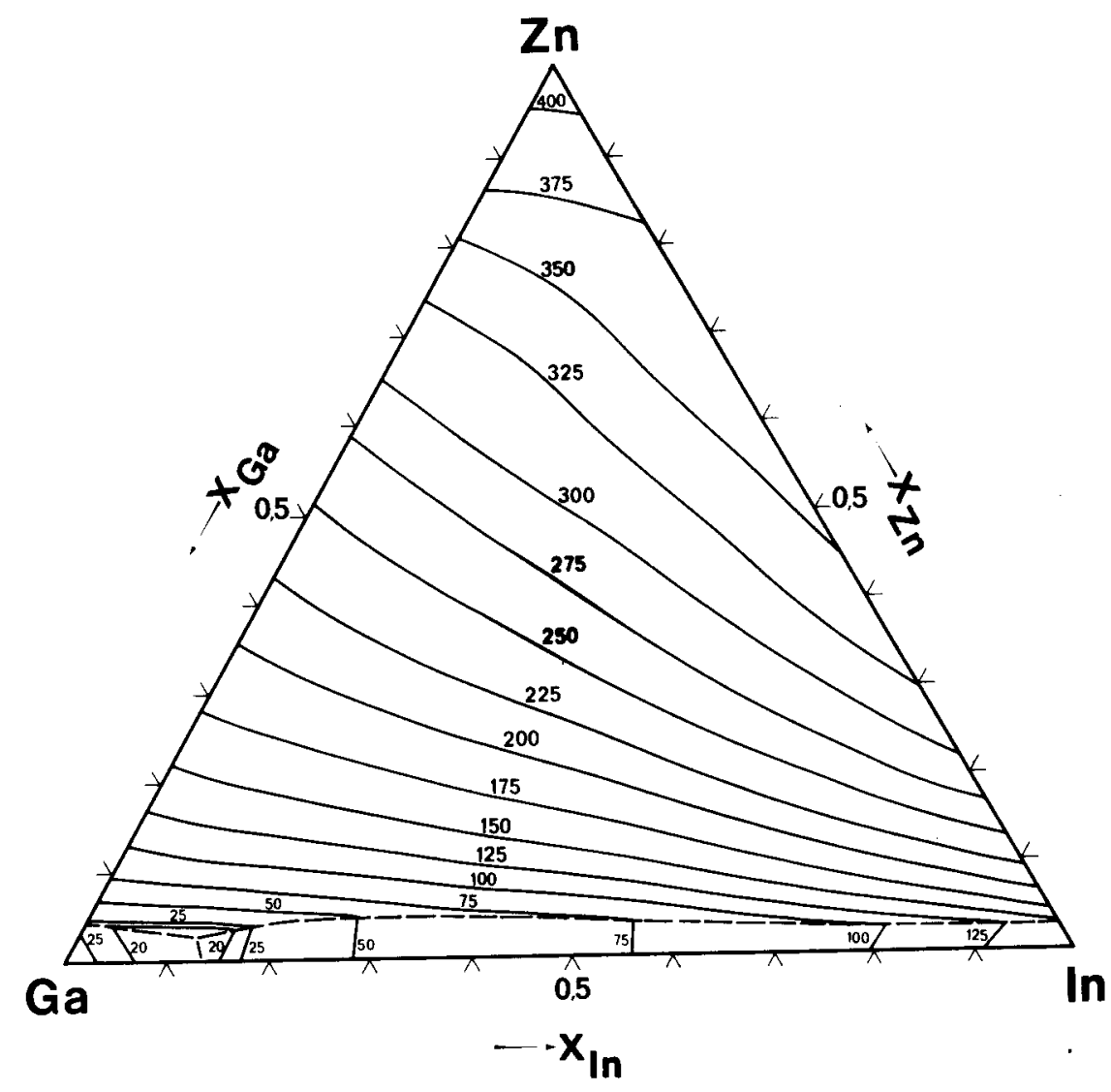


PARTIAL AND INTEGRAL HEATS OF MIXING FOR LIOUID - GA-IN-ZN SOLUTIONS AT 723K (CAL/MOL)

\begin{tabular}{|c|c|c|c|c|c|}
\hline$X \quad 2 N$ & $X G A / X I N$ & $\mathrm{DH} \quad \mathrm{ZN}$ & $\mathrm{DH} \quad \mathrm{GA}$ & $\mathrm{DH}$ IN & DH \\
\hline 0.0 & 9.000 & 1435 & 11 & 859 & 95 \\
\hline 0.1 & 9.000 & 1314 & 13 & 915 & 224 \\
\hline 0.2 & 9.000 & 1151 & 34 & 1019 & 336 \\
\hline 0.3 & 9.000 & 969 & 82 & 1194 & 426 \\
\hline 0.4 & 9.000 & 779 & $1 \circledast$ & 1439 & 490 \\
\hline 0.5 & 9.000 & 591 & 308 & 1747 & 521 \\
\hline 0.6 & 9.000 & 411 & 511 & 2115 & 515 \\
\hline 0.7 & 9.000 & 251 & 796 & 2542 & 467 \\
\hline 0.8 & 9.000 & 121 & 1179 & 3030 & 369 \\
\hline 0.9 & 9.000 & 33 & 1677 & 3583 & 216 \\
\hline $1 \cdot 0$ & 9.000 & 0 & 2311 & 4206 & 0 \\
\hline 0.0 & 4.000 & 1478 & 42 & 678 & 170 \\
\hline 0.1 & 4.000 & 1371 & 36 & 742 & 296 \\
\hline 0.2 & 4.000 & 1209 & 49 & 836 & 407 \\
\hline 0.3 & 4.000 & 1030 & 90 & 972 & 495 \\
\hline 0.4 & 4.000 & 834 & 172 & 1177 & 557 \\
\hline 0.5 & 4.000 & 632 & 308 & 1463 & 586 \\
\hline 0.6 & 4.000 & 438 & 513 & 1834 & 574 \\
\hline 0.7 & 4.000 & 265 & 803 & 2293 & 515 \\
\hline 0.8 & 4.000 & 126 & 1189 & 2841 & 404 \\
\hline 0.9 & 4.000 & 33 & 1688 & 3478 & 235 \\
\hline 1.0 & 4.000 & 0 & 2311 & 4206 & 0 \\
\hline 0.0 & 2.333 & 1543 & 95 & 519 & 223 \\
\hline 0.1 & 2.333 & 1443 & 77 & 588 & 351 \\
\hline 0.2 & 2.333 & 1266 & 78 & 689 & 462 \\
\hline 0.3 & 2.333 & 1080 & 112 & 819 & 551 \\
\hline 0.4 & 2.333 & 879 & 189 & 1003 & 612 \\
\hline 0.5 & 2.333 & 670 & 323 & 1265 & 638 \\
\hline 0.6 & 2.333 & 465 & 529 & 1624 & 622 \\
\hline 0.7 & 2.333 & 281 & 819 & 2091 & 557 \\
\hline 0.8 & 2.333 & 133 & 1206 & 2675 & 436 \\
\hline 0.9 & 2.333 & 35 & 1700 & 3379 & 252 \\
\hline $1 \cdot 0$ & 2.333 & 0 & 2311 & 4206 & 0 \\
\hline 0.0 & 1.500 & 1629 & 170 & 382 & 254 \\
\hline 0.1 & 1.500 & 1530 & 135 & 452 & 389 \\
\hline 0.2 & 1.500 & 1334 & 123 & 558 & 504 \\
\hline 0.3 & 1.500 & 1130 & 147 & 692 & 595 \\
\hline 0.4 & 1.500 & 919 & 220 & 870 & 655 \\
\hline 0.5 & 1.500 & 703 & 352 & 1117 & $\circledast 0$ \\
\hline 0.6 & 1.500 & 491 & 557 & 1461 & 662 \\
\hline 0.7 & 1.500 & 298 & 846 & 1926 & 592 \\
\hline 0.8 & 1.500 & 142 & 1229 & 2530 & 463 \\
\hline 0.9 & 1.500 & 38 & 1715 & 3287 & $2 \theta 8$ \\
\hline 1.0 & 1.500 & 0 & 2311 & 4206 & 0 \\
\hline
\end{tabular}




\begin{tabular}{|c|c|c|c|c|c|}
\hline 0.0 & 1.000 & 1736 & 265 & 265 & 265 \\
\hline 0.1 & 1.000 & 1630 & 211 & 335 & 408 \\
\hline 0.2 & 1.000 & 1414 & 182 & 441 & 532 \\
\hline 0.3 & 1.000 & 1185 & 196 & 581 & 627 \\
\hline 0.4 & 1.000 & 957 & 264 & 759 & 60 \\
\hline 0.5 & 1.000 & 732 & 394 & 999 & 74 \\
\hline 0.6 & 1.000 & 515 & 598 & 1331 & 694 \\
\hline 0.7 & 1.000 & 316 & 882 & 1788 & 622 \\
\hline 0.8 & 1.000 & 152 & 1257 & 2401 & 488 \\
\hline 0.9 & 1.000 & 41 & 1731 & 3200 & 283 \\
\hline $1 \cdot 0$ & 1.000 & 0 & 2311 & 4206 & 0 \\
\hline 0.0 & 0.667 & 1864 & 382 & 170 & 254 \\
\hline 0.1 & 0.667 & 1745 & 304 & 235 & 411 \\
\hline 0.2 & 0.667 & 1504 & 257 & 339 & 546 \\
\hline 0.3 & 0.667 & 1245 & 260 & 481 & 649 \\
\hline 0.4 & 0.667 & 997 & 323 & 663 & 715 \\
\hline 0.5 & 0.667 & 760 & 452 & 901 & 741 \\
\hline 0.6 & 0.667 & 538 & 651 & 1224 & 721 \\
\hline 0.7 & 0.667 & 334 & 929 & 1672 & 646 \\
\hline 0.8 & 0.667 & 164 & 1292 & 2288 & 509 \\
\hline 0.9 & 0.667 & 45 & 1749 & 3118 & 298 \\
\hline $1 \cdot 0$ & 0.667 & 0 & 2311 & 4206 & 0 \\
\hline 0.0 & 4.286 & 1473 & 38 & 697 & 163 \\
\hline 0.1 & $4 \cdot 286$ & 1364 & 33 & 760 & 290 \\
\hline 0.2 & 4.286 & 1203 & 46 & 853 & 400 \\
\hline 0.3 & 4.286 & 1024 & 88 & 992 & 489 \\
\hline 0.4 & 4.286 & 829 & 170 & 1200 & 551 \\
\hline 0.5 & 4.286 & 628 & 307 & 1488 & 579 \\
\hline 0.6 & 4.286 & 435 & 513 & 1861 & 568 \\
\hline 0.7 & 4.286 & 263 & 801 & 2318 & 511 \\
\hline 0.8 & 4.286 & 125 & 1188 & 2860 & 401 \\
\hline 0.9 & 4.286 & 33 & 1687 & 3489 & 233 \\
\hline 1.0 & 4.286 & 0 & 2311 & 4206 & 0 \\
\hline 0.0 & 2.500 & 1532 & 87 & 541 & 216 \\
\hline 0.1 & 2.500 & 1432 & $\pi 0$ & 609 & 345 \\
\hline 0.2 & 2.500 & 1258 & 73 & 709 & 455 \\
\hline 0.3 & 2.500 & 1073 & 108 & 839 & 544 \\
\hline 0.4 & $2 \cdot 500$ & 873 & 186 & 1025 & 605 \\
\hline 0.5 & 2.500 & 665 & 320 & 1290 & 631 \\
\hline 0.6 & 2.500 & 461 & 526 & 1651 & 616 \\
\hline 0.7 & 2.500 & 278 & 816 & 2118 & 551 \\
\hline 0.8 & 2.500 & 132 & 1203 & 2698 & 431 \\
\hline 0.9 & 2.500 & 35 & 1698 & 3393 & 250 \\
\hline $1 \cdot 0$ & 2.500 & 0 & 2311 & 4206 & 0 \\
\hline 0.0 & 0.111 & 2375 & 859 & 11 & 95 \\
\hline 0.1 & 0.111 & 2157 & 200 & 44 & 314 \\
\hline 0.2 & 0.111 & 1808 & 614 & 123 & 499 \\
\hline 0.3 & 0.111 & 1450 & 598 & 257 & 639 \\
\hline 0.4 & 0.111 & 1128 & 646 & 445 & 730 \\
\hline 0.5 & 0.111 & 850 & 752 & 686 & 771 \\
\hline 0.6 & 0.111 & 608 & 915 & 998 & 761 \\
\hline 0.7 & 0.111 & 393 & 1139 & 1419 & 692 \\
\hline 0.8 & 0.111 & 206 & 1433 & 2018 & 556 \\
\hline $\begin{array}{l}0.9 \\
1.0\end{array}$ & 0.111 & 61 & 1815 & 2900 & 334 \\
\hline $1 \cdot 0$ & 0.111 & 0 & 2311 & 4206 & 0 \\
\hline
\end{tabular}


PARTIAL AND INTEGRAL EXCESS ENTROPIES OF MIXING FOR LIQUID GA-IN-ZN SOLUTIONS AT 723K (CAL/MOL)/K

\begin{tabular}{|c|c|c|c|c|c|}
\hline$X \quad \mathrm{ZN}$ & $X G A / X I N$ & SXS ZN & SXS GA & SXS IN & sXs \\
\hline 0.0 & 9.000 & $2 \cdot 3825$ & -0.0023 & -0.2599 & -0.0280 \\
\hline 0.1 & 9.000 & 1.3968 & 0.0515 & -0.3014 & 0.1543 \\
\hline 0.2 & 9.000 & 1.0489 & 0.1114 & -0.2656 & 0.2688 \\
\hline 0.3 & 9.000 & 0.9092 & 0.1549 & -0.1988 & 0.3565 \\
\hline 0.4 & 9.000 & 0.7686 & 0.2309 & -0.1112 & 0.4255 \\
\hline 0.5 & 9.000 & 0.5681 & 0.4030 & 0.0062 & 0.4657 \\
\hline 0.6 & 9.000 & 0.3350 & 0.2040 & 0.1691 & 0.4612 \\
\hline 0.7 & 9.000 & 0.1307 & 1.1010 & 0.3888 & 0.4004 \\
\hline 0.8 & 9.000 & 0.0090 & $1 \cdot 4684$ & 0.6635 & 0.2848 \\
\hline 0.9 & 9.000 & -0.0169 & 1.5685 & 0.9720 & 0.1357 \\
\hline $1 \cdot 0$ & 9.000 & -0.0000 & 1.0375 & 1.2672 & 0.0000 \\
\hline 0.0 & 4.000 & $2 \cdot 2631$ & -0.0072 & -0.2312 & -0.0520 \\
\hline 0.1 & 4.000 & 1.3463 & 0.0526 & -0.2699 & 0.1239 \\
\hline 0.2 & 4.000 & 1.0536 & 0.1058 & -0.2411 & 0.2398 \\
\hline 0.3 & 4.000 & 0.9200 & 0.1468 & -0.1833 & 0.3325 \\
\hline 0.4 & 4.000 & 0.7590 & 0.2368 & -0.1014 & 0.4051 \\
\hline 0.5 & 4.000 & 0.5408 & 0.4336 & 0.0155 & 0.4454 \\
\hline 0.6 & 4.000 & 0.3074 & 0.7508 & 0.1808 & 0.4391 \\
\hline 0.7 & 4.000 & 0.1173 & $1 \cdot 1355$ & 0.4017 & 0.3787 \\
\hline 0.8 & 4.000 & 0.0105 & 1.4598 & 0.6731 & 0.2689 \\
\hline 0.9 & 4.000 & -0.0123 & 1.5193 & 0.9743 & 0.1300 \\
\hline 1.0 & 4.000 & -0.0000 & 1.0375 & 1.2672 & 0.0000 \\
\hline 0.0 & 2.333 & 2.1406 & -0.0145 & -0.2096 & -0.0730 \\
\hline 0.1 & 2.333 & $1 \cdot 2969$ & 0.0508 & -0.2427 & 0.0962 \\
\hline 0.2 & 2.333 & 1.0575 & $0.09 \circledast$ & -0.2183 & 0.2133 \\
\hline 0.3 & 2.333 & 0.9231 & 0.1398 & -0.1678 & 0.3102 \\
\hline 0.4 & 2.333 & 0.7397 & 0.2502 & -0.0901 & 0.3848 \\
\hline 0.5 & 2.333 & 0.5080 & 0.4736 & 0.0269 & 0.4238 \\
\hline 0.6 & 2.333 & 0.2801 & 0.8009 & 0.1944 & 0.4156 \\
\hline 0.7 & 2.333 & $0.10 \circledast 8$ & $1 \cdot 1635$ & 0.4152 & 0.3565 \\
\hline 0.8 & 2.333 & 0.0137 & 1.4393 & 0.6819 & 0.2534 \\
\hline 0.9 & 2.333 & -0.0076 & 1.4629 & 0.9758 & 0.1248 \\
\hline $1 \cdot 0$ & 2.333 & -0.0000 & 1.0375 & 1.2672 & 0.0000 \\
\hline 0.0 & 1.500 & 2.0150 & -0.0266 & -0.1873 & -0.0909 \\
\hline 0.1 & 1.500 & $1 \cdot 2518$ & 0.0431 & -0.2143 & 0.0713 \\
\hline 0.2 & 1.500 & 1.0600 & 0.0823 & -0.1934 & 0.1896 \\
\hline 0.3 & 1.500 & 0.9158 & 0.1344 & -0.1488 & 0.2895 \\
\hline 0.4 & 1.500 & 0.7095 & 0.2725 & -0.0738 & 0.3642 \\
\hline 0.5 & 1.500 & 0.4707 & 0.5217 & 0.0440 & 0.4007 \\
\hline 0.6 & 1.500 & 0.2545 & 0.8500 & 0.2123 & 0.3907 \\
\hline 0.7 & 1.500 & 0.0999 & 1.1795 & 0.4310 & 0.3340 \\
\hline 0.8 & 1.500 & 0.0187 & 1.4032 & 0.6912 & 0.2387 \\
\hline 0.9 & 1.500 & -0.0030 & $1 \cdot 3984$ & 0.9772 & 0.1203 \\
\hline $1 \cdot 0$ & 1.500 & -0.0000 & 1.0375 & 1.2672 & 0.0000 \\
\hline
\end{tabular}




\begin{tabular}{|c|c|c|c|c|c|}
\hline 0.0 & 1.000 & 1.8853 & -0.0495 & -0.1597 & -0.1046 \\
\hline $0 \cdot 1$ & 1.000 & $1 \cdot 2126$ & 0.0236 & -0.1812 & 0.0503 \\
\hline 0.2 & 1.000 & 1.0579 & 0.0587 & -0.1638 & 0.1696 \\
\hline 0.3 & 1.000 & 0.8939 & 0.1307 & -0.1236 & 0.2707 \\
\hline 0.4 & 1.000 & 0.6677 & 0.3035 & -0.0497 & 0.3432 \\
\hline 0.5 & 1.000 & 0.4308 & 0.5740 & 0.0688 & 0.3761 \\
\hline 0.6 & 1.000 & 0.2325 & 0.8901 & 0.2364 & 0.3648 \\
\hline 0.7 & 1.000 & 0.0971 & 1.1754 & 0.4501 & 0.3118 \\
\hline 0.8 & 1.000 & 0.0253 & $1 \cdot 3467$ & 0.7017 & 0.2251 \\
\hline 0.9 & 1.000 & 0.0014 & $1 \cdot 3244$ & 0.9789 & 0.1164 \\
\hline $1 \cdot 0$ & 1.000 & -0.0000 & 1.0375 & 1.2672 & 0.0000 \\
\hline 0.0 & 0.667 & $1 \cdot 7493$ & -0.0921 & -0.1253 & -0.1120 \\
\hline 0.1 & 0.667 & 1.1796 & -0.0167 & -0.1420 & 0.0352 \\
\hline 0.2 & 0.667 & 1.0445 & 0.0223 & -0.1282 & 0.1545 \\
\hline 0.3 & 0.667 & 0.8527 & 0.1294 & -0.0905 & 0.2540 \\
\hline 0.4 & 0.667 & 0.6149 & 0.3406 & -0.0163 & 0.3218 \\
\hline 0.5 & 0.667 & 0.3911 & 0.6206 & 0.1025 & 0.3504 \\
\hline 0.6 & 0.667 & 0.2158 & 0.9077 & 0.2663 & 0.3388 \\
\hline 0.7 & 0.667 & 0.0985 & $1 \cdot 1396$ & 0.4727 & 0.2908 \\
\hline 0.8 & 0.667 & 0.0329 & $1 \cdot 2636$ & 0.7135 & 0.2131 \\
\hline 0.9 & 0.667 & 0.0055 & 1.2393 & 0.9812 & 0.1134 \\
\hline $1 \cdot 0$ & 0.667 & 0.0000 & 1.0375 & 1.2672 & 0.0000 \\
\hline 0.0 & 4.286 & $2 \cdot 2761$ & -0.0066 & -0.2338 & -0.0496 \\
\hline 0.1 & 4.286 & 1.3517 & 0.0526 & -0.2730 & 0.1271 \\
\hline 0.2 & $4 \cdot 286$ & 1.0531 & 0.1065 & -0.2437 & 0.2428 \\
\hline 0.3 & 4.286 & 0.9191 & 0.1476 & -0.1850 & 0.3350 \\
\hline 0.4 & 4.286 & 0.7605 & 0.2358 & -0.1025 & 0.4073 \\
\hline 0.5 & $4 \cdot 286$ & 0.5440 & 0.4299 & 0.0144 & 0.4476 \\
\hline 0.6 & $4 \cdot 286$ & 0.3104 & 0.7455 & 0.1795 & 0.4416 \\
\hline 0.7 & $4 \cdot 286$ & 0.1186 & 1.1320 & 0.4003 & 0.3811 \\
\hline 0.8 & $4 \cdot 286$ & 0.0102 & $1 \cdot 4613$ & 0.6721 & 0.2706 \\
\hline 0.9 & $4 \cdot 286$ & -0.0128 & 1.5249 & 0.9741 & 0.1306 \\
\hline $1 \cdot 0$ & $4 \cdot 286$ & -0.0000 & 1.0375 & 1.2672 & 0.0000 \\
\hline 0.0 & 2.500 & 2.1583 & -0.0132 & -0.2126 & -0.0702 \\
\hline 0.1 & 2.500 & $1 \cdot 3038$ & 0.0513 & -0.2465 & 0.1000 \\
\hline 0.2 & 2.500 & 1.0570 & 0.0983 & -0.2216 & 0.2169 \\
\hline 0.3 & 2.500 & 0.9232 & 0.1407 & -0.1702 & 0.3133 \\
\hline 0.4 & 2.500 & 0.7431 & 0.2478 & -0.0920 & 0.3877 \\
\hline 0.5 & 2.500 & 0.5130 & 0.4673 & 0.0250 & 0.4270 \\
\hline 0.6 & 2.500 & 0.2839 & 0.7936 & 0.1922 & 0.4191 \\
\hline $0 \cdot 7$ & 2.500 & 0.1081 & 1.1601 & 0.4131 & 0.3597 \\
\hline 0.8 & 2.500 & 0.0131 & $1 \cdot 4431$ & 0.6807 & 0.2556 \\
\hline 0.9 & 2.500 & -0.0083 & 1.4714 & 0.9756 & 0.1255 \\
\hline $1 \cdot 0$ & 2.500 & -0.0000 & 1.0375 & 1.2672 & 0.0000 \\
\hline 0.0 & 0.111 & $1 \cdot 2667$ & -0.4741 & -0.0137 & -0.0598 \\
\hline $0 \cdot 1$ & 0.111 & 1.0326 & -0.3609 & $-0.016 B$ & 0.0572 \\
\hline 0.2 & 0.111 & 0.8173 & -0.1511 & 0.0026 & 0.1532 \\
\hline 0.3 & 0.111 & 0.6221 & 0.0644 & 0.0508 & 0.2232 \\
\hline 0.4 & 0.111 & 0.4586 & 0.2448 & 0.1285 & 0.2675 \\
\hline 0.5 & 0.111 & 0.3217 & 0.3946 & 0.2363 & 0.2869 \\
\hline $0 \cdot 6$ & 0.111 & 0.2089 & 0.5253 & 0.3751 & 0.2814 \\
\hline 0.7 & 0.111 & 0.1195 & 0.6475 & 0.5462 & 0.2505 \\
\hline 0.8 & 0.111 & 0.0541 & 0.7695 & 0.7509 & 0.1938 \\
\hline 0.9 & 0.111 & 0.0138 & 0.8979 & 0.9908 & 0.1105 \\
\hline $1 \cdot 0$ & 0.111 & 0.0000 & 1.0375 & 1.2672 & 0.0000 \\
\hline
\end{tabular}




\section{BIBLIOGRAPHY}

(1) M. GAMBINO, J.P. BROS and I. ANSARA, "Thermochimie", Coll. Int. CNRS $N^{\circ} 201,1971$, Marseille, p. 273.

(2) W.J. SVIRBELY and S.M. READ, J. Phys. Chem., 1962, 66, 4, 658 .

(3) F.H. HAYES and O. KUBASCHEWSKI, J. Inst. Metal., 1969, 97, 381.

(4) R. HULtGRen, P.D. DESAi, D.T. HAWKins, M. GLeiser, K.K. KELLEY, "Selected Values of the Thermodynamic Properties of Binary Alloys", 1973, American Soc. for Metals, Metals Park, Ohio, USA 


\section{I - EXPERIMENTAL RESULTS.-}

The heats of mixing of $\mathrm{Cu}-\mathrm{Fe}-\mathrm{Ni}$ solid solutions have been determined with an adiabatic calorimeter at $1323 \mathrm{~K}$ for nine different alloy compositions given in table $I$.

This ternary system exhibits a miscibility gap in the solid state which has been determined by BRADLEY ${ }^{(2)}$ at 1123 and $1323 \mathrm{~K}$.

\section{II - COMPARISON BETWEEN EXPERIMENTAL AND CALCULATED THERMODYNAMIC}

DATA. -

$$
\begin{aligned}
& \text { a) Binary solutions : } \\
& \mathrm{Cu}-\mathrm{Ni}(3)\left\{\begin{array}{l}
\Delta \mathrm{H}_{\mathrm{M}}=\mathrm{x}_{\mathrm{Cu}} \mathrm{x}_{\mathrm{Ni}}\left[857 \mathrm{x}_{\mathrm{Cu}}+3092 \mathrm{x}_{\mathrm{Ni}}\right] \\
\Delta \mathrm{S}^{\mathrm{xS}}=\mathrm{x}_{\mathrm{Cu}} \mathrm{x}_{\mathrm{Ni}}\left[-1.1642 \mathrm{x}_{\mathrm{Cu}}-0.4970 \mathrm{x}_{\mathrm{Ni}}\right]
\end{array}\right. \\
& \mathrm{Fe}-\mathrm{Ni}(4)\left\{\begin{array}{l}
\Delta \mathrm{H}_{\mathrm{M}}=\mathrm{x}_{\mathrm{Fe}} \mathrm{x}_{\mathrm{Ni}}\left[-6642 \mathrm{x}_{\mathrm{Ni}}-483 \mathrm{x}_{\mathrm{Fe}}\right] \\
\Delta \mathrm{S}^{\mathrm{XS}}=\mathrm{x}_{\mathrm{Fe}} \mathrm{x}_{\mathrm{Ni}}\left[-3.1295 \mathrm{x}_{\mathrm{Ni}}+0.4612 \mathrm{x}_{\mathrm{Fe}}\right]
\end{array}\right. \\
& \mathrm{Cu}-\mathrm{Fe}(1)\left\{\begin{array}{l}
{ }_{\mathrm{H}} \mathrm{H}_{\mathrm{M}}=\mathrm{x}_{\mathrm{Cu}} \mathrm{x}_{\mathrm{Fe}}\left[12936 \mathrm{x}_{\mathrm{Fe}}+10107 \mathrm{x}_{\mathrm{Cu}}\right] \\
\text { (derived from the heats of mixing of the ternary } \\
\text { solid solutions) } \\
\Delta \mathrm{S}^{\mathrm{XS}}=\mathrm{x}_{\mathrm{Cu}} \mathrm{x}_{\mathrm{Fe}}^{\left[2.9805 \mathrm{x}_{\mathrm{Cu}}+2.9805 \mathrm{x}_{\mathrm{Fe}}\right]} \\
\text { (derived from the bj nary phase diagram using the } \\
\text { extrapolated values of } \Delta \mathrm{H}_{\mathrm{M}} \text { ) }
\end{array}\right.
\end{aligned}
$$

\section{b) Ternary solutions :}

1) Heats of mixing :

The experimental values of the heats of mixing were fitted by the following equation :

$$
\begin{aligned}
{ }_{\mathrm{H}} & =\mathrm{x}_{\mathrm{Cu}} \mathrm{x}_{\mathrm{Ni}}\left[857 \mathrm{x}_{\mathrm{Cu}}+3092 \mathrm{x}_{\mathrm{Ni}}\right]+\mathrm{x}_{\mathrm{Fe}} \mathrm{x}_{\mathrm{Ni}}\left[-6642 \mathrm{x}_{\mathrm{Ni}}-483 \mathrm{x}_{\mathrm{Fe}}\right] \\
& +\mathrm{x}_{\mathrm{Cu}} \mathrm{x}_{\mathrm{Fe}}\left[\mathrm{Ax} \mathrm{x}_{\mathrm{Fe}}+\mathrm{B} \mathrm{x}_{\mathrm{Cu}}\right]+\mathrm{C} \mathrm{x}_{\mathrm{Cu}} \mathrm{x}_{\mathrm{Fe}}{ }^{\mathrm{x}} \mathrm{Ni}
\end{aligned}
$$


which yields : $A=12936 ; \quad B=10107 ; \quad C=-2066^{\circ}$

Table I shows that the calculated values are within the experimental error ( $\pm 150 \mathrm{cal}^{\mathrm{mol}}{ }^{-1}$ ) except for one point.

\section{Table I}

$\begin{array}{lcccc}x_{\mathrm{Cu}} & \mathrm{x}_{\mathrm{Ni}} & \mathrm{x}_{\mathrm{Fe}} & \exp & \exp -\mathrm{calc} . \\ 0.050 & 0.900 & 0.050 & -283 & -139 \\ 0.100 & 0.800 & 0.100 & -250 & -33 \\ 0.050 & 0.760 & 0.190 & -710 & -73 \\ 0.190 & 0.760 & 0.050 & 492 & -313 \\ 0.167 & 0.667 & 0.167 & -330 & -143 \\ 0.200 & 0.600 & 0.200 & -51 & 60 \\ 0.110 & 0.445 & 0.445 & -245 & 19 \\ 0.445 & 0.445 & 0.110 & 434 & -11 \\ 0.333 & 0.333 & 0.333 & 653 & -\end{array}$

2) Excess entropy of mixing :

The excess entropy of mixing is calculated from the binary data by the following equation :

$$
\begin{aligned}
& \Delta \mathrm{S}_{\mathrm{M}}^{\mathrm{xS}}=\mathrm{x}_{\mathrm{Cu}} \mathrm{x}_{\mathrm{Ni}}\left[-1.1642 \mathrm{x}_{\mathrm{Cu}}-0.4970 \mathrm{x}_{\mathrm{Ni}}\right]+\mathrm{x}_{\mathrm{Fe}} \mathrm{x}_{\mathrm{Ni}}\left[-3.1295 \mathrm{x}_{\mathrm{Ni}}+0.4612 \mathrm{x}_{\mathrm{Fe}}\right] \\
& +x_{C u} x_{F e}\left[A^{\prime} x_{C u}+A^{\prime} x_{F e}\right] \\
& A^{\prime}=2.9805 \text { (derived from the binary phase diagram using the } \\
& \text { extrapolated values of } \Delta \mathrm{H}_{M}{ }^{\prime} \text {. }
\end{aligned}
$$

III - COMPARISON WITH THE EXPERIMENTAL PHASE DIAGRAM .-

The isothermal sections at 1123 and $1323 \mathrm{~K}$ are calculated ${ }^{(1)}$ using the above data for the ternary solution and compared to the experimental results in figure 1. A satisfactory agreement is observed. 


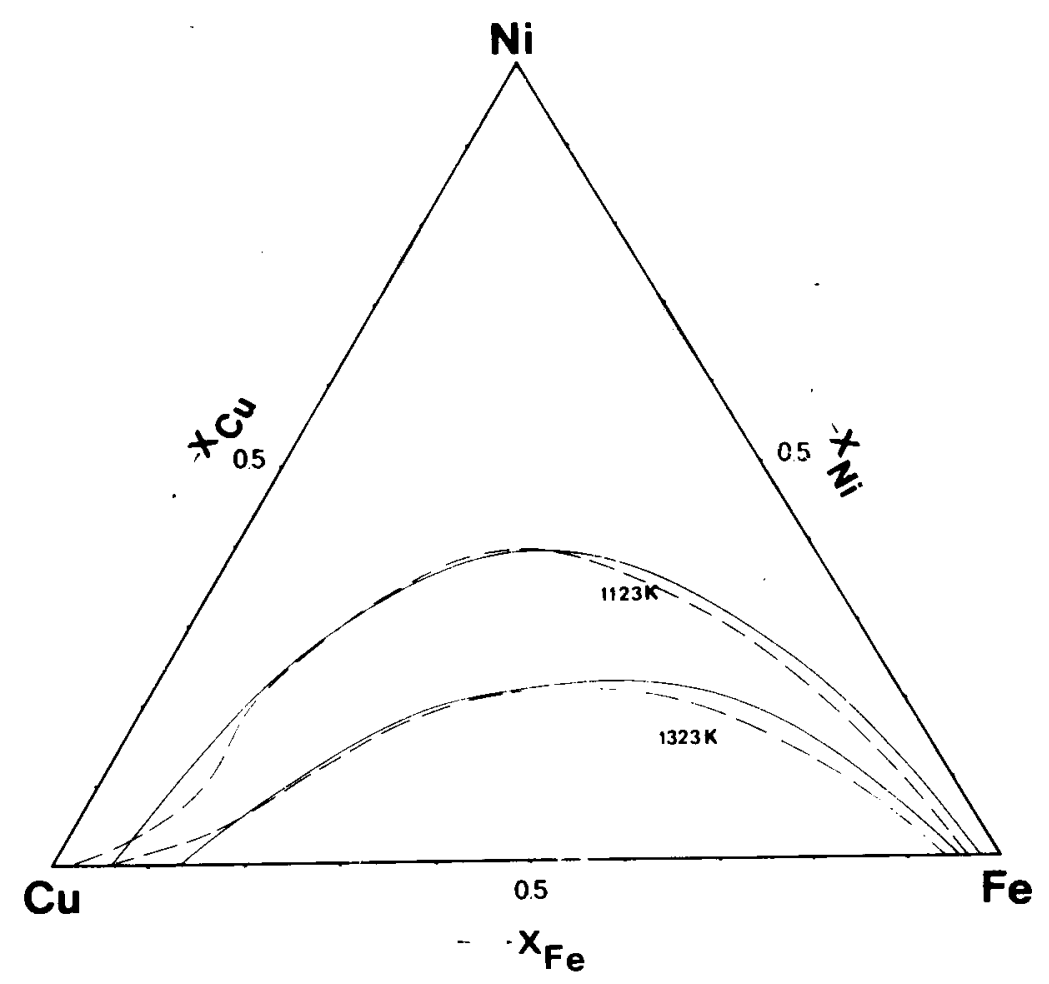


PAKTIAL AND INTEGRAL HEATS OF MIXING FOK SOLID

HOMOGENEOUS CU-FE-NI SOLUIIIONS A'I 1323K (CAL/MOL)

\begin{tabular}{|c|c|c|c|c|c|}
\hline X NI & $X \mathrm{CU} / \mathrm{XFE}$ & $\mathrm{DH} \mathrm{NI}$ & $\mathrm{DH} \quad \mathrm{CU}$ & $\mathrm{DH} \quad \mathrm{FE}$ & $\mathrm{DH}$ \\
\hline 0.0 & 9.000 & -1367 & 78 & 8645 & 935 \\
\hline 0.1 & 9.000 & -694 & 235 & 6790 & 742 \\
\hline 0.2 & 9.000 & -221 & 349 & 4916 & 611 \\
\hline 0.3 & 9.000 & 82 & 448 & 3057 & 528 \\
\hline 0.4 & 9.000 & 246 & 560 & 1248 & 478 \\
\hline 0.5 & 9.000 & 299 & 714 & -478 & 445 \\
\hline 0.6 & 9.000 & 274 & 936 & -2086 & 413 \\
\hline 0.7 & 9.000 & 201 & 1257 & -3541 & 368 \\
\hline 0.8 & 9.000 & 110 & 1704 & -4809 & 295 \\
\hline 0.9 & 9.000 & 33 & 2306 & -5854 & 177 \\
\hline $1 \cdot 0$ & 9.000 & 0 & 3092 & -6642 & 0 \\
\hline 0.0 & 4.000 & -3217 & 336 & 7193 & 1708 \\
\hline 0.1 & 4.000 & -2175 & 598 & 5813 & 127 \\
\hline 0.2 & 4.000 & -1374 & 291 & 4331 & 936 \\
\hline 0.3 & 4.000 & -787 & 943 & 2781 & 687 \\
\hline 0.4 & 4.000 & -381 & 1077 & 1204 & 507 \\
\hline 0.5 & 4.000 & -126 & 1223 & -363 & 381 \\
\hline 0.6 & 4.000 & 9 & 1406 & -1880 & 293 \\
\hline 0.7 & 4.000 & 56 & 1656 & -3307 & 227 \\
\hline 0.8 & 4.000 & 47 & 2000 & -4604 & 167 \\
\hline 0.9 & 4.000 & 18 & 2469 & -5730 & 96 \\
\hline $1 \cdot 0$ & 4.000 & 0 & 3092 & -6642 & 0 \\
\hline 0.0 & 2.333 & -4659 & 808 & 5784 & 2301 \\
\hline 0.1 & 2.333 & -3362 & 1104 & 4835 & 1674 \\
\hline 0.2 & 2.333 & -2323 & 1325 & 3716 & 1176 \\
\hline 0.3 & 2.333 & -1518 & 1491 & 2463 & 792 \\
\hline 0.4 & 2.333 & -919 & 1624 & 1116 & 505 \\
\hline 0.5 & 2.333 & -499 & 1746 & -287 & 302 \\
\hline 0.6 & 2.333 & -229 & 1881 & -1705 & 166 \\
\hline 0.7 & 2.33 .3 & -77 & 2056 & -3093 & 84 \\
\hline 0.8 & 2.333 & -10 & 2296 & -4410 & 39 \\
\hline 0.9 & 2.333 & 3 & 2632 & -5608 & 16 \\
\hline $1 \cdot 0$ & 2.333 & 0 & 3092 & -6642 & 0 \\
\hline 0.0 & 1.500 & -5659 & 1527 & 4453 & 2697 \\
\hline 0.1 & $1 \cdot 500$ & -4229 & 1779 & 3880 & 1938 \\
\hline 0.2 & 1.500 & -3048 & 1968 & 3090 & 1321 \\
\hline 0.3 & 1.500 & -2099 & 2107 & 2115 & 836 \\
\hline 0.4 & 1.500 & -1362 & 2208 & 990 & 468 \\
\hline 0.5 & 1.500 & -816 & 2287 & -248 & 204 \\
\hline 0.6 & 1.500 & -436 & 2364 & -1558 & 32 \\
\hline 0.7 & 1.500 & -195 & 2457 & -2899 & -62 \\
\hline 0.8 & 1.500 & -63 & 2592 & -4225 & -89 \\
\hline 0.9 & 1.500 & -10 & 2795 & -5489 & -65 \\
\hline 1.0 & 1.500 & -0 & 3092 & -6642 & -0 \\
\hline
\end{tabular}




\begin{tabular}{|c|c|c|c|c|c|}
\hline 0.0 & 1.000 & -6184 & 2527 & 3234 & 2880 \\
\hline 0.1 & 1.000 & -4753 & 2646 & 2974 & 2050 \\
\hline $0 \cdot 2$ & 1.000 & -3533 & 2738 & 2471 & 1364 \\
\hline 0.3 & 1.000 & -2519 & 2801 & 1749 & 814 \\
\hline 0.4 & 1.000 & -1703 & 2837 & 835 & 391 \\
\hline 0.5 & 1.000 & -1071 & 2352 & -239 & 85 \\
\hline 0.6 & 1.000 & -610 & 2856 & -1438 & -112 \\
\hline 0.7 & 1.000 & -297 & 2862 & -2722 & -210 \\
\hline 0.8 & 1.000 & -111 & 2889 & -4050 & -218 \\
\hline 0.9 & 1.000 & -22 & 2957 & -5373 & -145 \\
\hline $1 \cdot 0$ & 1.000 & -0 & 3092 & -6642 & -0 \\
\hline 0.0 & 0.667 & -6199 & 3843 & 2160 & 2833 \\
\hline 0.1 & 0.667 & -4908 & 3732 & 2140 & 1997 \\
\hline 0.2 & 0.667 & -3760 & 3653 & 1876 & 1294 \\
\hline 0.3 & 0.667 & -2765 & 3586 & 1376 & 720 \\
\hline 0.4 & 0.667 & -1932 & 3519 & 658 & 271 \\
\hline 0.5 & 0.667 & -1261 & 3444 & -257 & -56 \\
\hline 0.6 & 0.667 & -748 & 3360 & -1341 & -266 \\
\hline 0.7 & $0.66^{7}$ & -384 & 3271 & -2563 & -361 \\
\hline 0.8 & 0.667 & -152 & 3186 & -3883 & -346 \\
\hline 0.9 & 0.667 & -33 & 3119 & -5259 & -225 \\
\hline $1 \cdot 0$ & $0.66 ?$ & -0 & 3092 & -6642 & -0 \\
\hline 0.0 & 0.429 & -5670 & 5507 & 1266 & 2538 \\
\hline 0.1 & 0.429 & -4669 & 5061 & 1405 & 1769 \\
\hline 0.2 & 0.429 & -3710 & 4729 & 1322 & 1105 \\
\hline 0.3 & 0.429 & -2826 & 4473 & 1009 & 549 \\
\hline 0.4 & 0.429 & -2043 & 4260 & 467 & 106 \\
\hline 0.5 & 0.429 & -1381 & $406 ?$ & -297 & -222 \\
\hline 0.6 & 0.429 & -849 & 3879 & -1266 & -430 \\
\hline 0.7 & 0.429 & -453 & 3685 & -2420 & -516 \\
\hline 0.8 & 0.429 & -188 & 3484 & -3726 & -475 \\
\hline 0.9 & 0.429 & -43 & 3282 & -5149 & -304 \\
\hline 1.0 & 0.429 & -0 & 3092 & -6642 & -0 \\
\hline 0.0 & 0.250 & -4564 & 7555 & 585 & 1979 \\
\hline 0.1 & 0.250 & -4011 & 6653 & 794 & 1352 \\
\hline 0.2 & 0.250 & -3367 & 5986 & 828 & 786 \\
\hline 0.3 & 0.250 & -2689 & 5474 & 660 & 294 \\
\hline 0.4 & 0.250 & -2029 & 5069 & 269 & -110 \\
\hline 0.5 & 0.250 & -1426 & 4727 & -354 & -415 \\
\hline 0.6 & 0.250 & -911 & 4414 & -1211 & -608 \\
\hline 0.7 & 0.250 & -504 & 4104 & -2292 & -675 \\
\hline 0.8 & 0.250 & -217 & 3783 & -3578 & -605 \\
\hline 0.9 & 0.250 & -52 & 3444 & -5040 & -384 \\
\hline $1 \cdot 0$ & 0.250 & -0 & 3092 & -6642 & -0 \\
\hline 0.0 & 0.111 & -2846 & 10020 & 152 & 1139 \\
\hline 0.1 & 0.111 & -2909 & 8549 & 330 & 733 \\
\hline 0.2 & 0.111 & -2712 & 7440 & 411 & 329 \\
\hline 0.3 & 0.111 & -2343 & 6602 & 340 & -50 \\
\hline 0.4 & 0.111 & -1881 & 5954 & 72 & -380 \\
\hline 0.5 & 0.111 & -1392 & 5428 & -426 & -637 \\
\hline 0.6 & 0.111 & -930 & 4968 & -1173 & -798 \\
\hline 0.7 & 0.111 & -536 & 4530 & -2178 & -838 \\
\hline 0.8 & 0.111 & -240 & 4083 & -3437 & -735 \\
\hline 0.9 & 0.111 & -59 & 3606 & -4935 & -463 \\
\hline $1 \cdot 0$ & 0.111 & -0 & 3092 & -6642 & -0 \\
\hline
\end{tabular}


PARTIAL AND INTEGRAL EXCESS ENTROPIES OF MIXING FOR HOMOGENEOUS SOLID CU-FE-NI SOLUTIONS AT 1323K (CAL/MOL)/K

\begin{tabular}{|c|c|c|c|c|c|}
\hline$X N I$ & $X C U / X F E$ & SX S N I & SXS CU & SXS FE & SXS \\
\hline 0.0 & 9.000 & -1.4749 & 0.0298 & 2.4142 & 0.2682 \\
\hline 0.1 & 9.000 & -1.1217 & 0.0403 & 2.1357 & 0.1127 \\
\hline 0.2 & 9.000 & -0.8283 & 0.0228 & 1.7768 & -0.007 \\
\hline 0.3 & 9.000 & -0.5894 & -0.0176 & 1.3445 & -0.0938 \\
\hline 0.4 & 9.000 & -0.4000 & -0.0758 & 0.8460 & -0.1502 \\
\hline 0.5 & 9.000 & -0.2546 & -0.1465 & 0.2884 & -0.1788 \\
\hline 0.6 & 9.000 & -0.1480 & -0.2242 & -0.3210 & -0.1824 \\
\hline 0.7 & 9.000 & -0.0748 & -0.3037 & -0.9750 & -0.1636 \\
\hline 0.8 & 9.000 & -0.0295 & -0.3794 & $-1 \cdot 6660$ & -0.1252 \\
\hline 0.9 & 9.000 & -0.0064 & -0.4457 & -2.3867 & -0.0698 \\
\hline $1 \cdot 0$ & 9.000 & -0.0000 & -0.4970 & -3.1295 & -0.0000 \\
\hline 0.0 & 4.000 & $-1 \cdot \notin 804$ & 0.1192 & 1.9075 & 0.4769 \\
\hline $\begin{array}{l}0.1 \\
0.2\end{array}$ & $\begin{array}{l}4.000 \\
4.000\end{array}$ & $\begin{array}{l}-1.3297 \\
-1.0242\end{array}$ & $\begin{array}{l}0.1396 \\
0.1372\end{array}$ & $\begin{array}{l}1.7336 \\
1.4715\end{array}$ & $\begin{array}{l}0.2796 \\
0.1184\end{array}$ \\
\hline 0.3 & 4.000 & -0.7626 & 0.1132 & 1.1263 & -0.0077 \\
\hline 0.4 & 4.000 & -0.5436 & 0.0690 & 0.7034 & -0.0999 \\
\hline 0.5 & 4.000 & -0.3652 & 0.0065 & 0.2083 & -0.1592 \\
\hline 0.6 & 4.000 & -0.2255 & -0.0722 & -0.3530 & -0.1866 \\
\hline 0.7 & 4.000 & -0.1219 & -0.1648 & -0.9741 & -0.1834 \\
\hline 0.8 & 4.000 & -0.0519 & -0.2685 & -1.6485 & -0.1504 \\
\hline 0.9 & 4.000 & -0.0124 & -0.3804 & -2.3694 & -0.0890 \\
\hline $1 \cdot 0$ & 4.000 & -0.0000 & -0.4970 & -3.1295 & -0.0000 \\
\hline 0.0 & 2.333 & $-1 \cdot 7808$ & 0.2682 & 1.4604 & 0.6259 \\
\hline 0.1 & 2.333 & -1.4590 & 0.2806 & 1.3740 & 0.4019 \\
\hline 0.2 & 2.333 & -1.1622 & 0.2803 & $1 \cdot 1962$ & 0.2116 \\
\hline 0.3 & 2.333 & -0.8941 & 0.2633 & 0.9289 & 0.0559 \\
\hline 0.4 & 2.333 & -0.6579 & 0.2267 & 0.5749 & -0.0645 \\
\hline 0.5 & 2.333 & -0.4562 & 0.1678 & 0.1376 & -0.1487 \\
\hline 0.6 & 2.333 & -0.2905 & 0.0849 & -0.3789 & -0.1960 \\
\hline 0.7 & 2.333 & -0.1621 & -0.0230 & -0.9697 & -0.2056 \\
\hline 0.8 & 2.333 & -0.0712 & -0.1564 & $-1 \cdot 6294$ & -0.1766 \\
\hline 0.9 & 2.333 & -0.0175 & -0.3147 & -2.3516 & -0.1084 \\
\hline $1 \cdot 0$ & 2.333 & -0.0000 & -0.4970 & -3.1295 & -0.0000 \\
\hline 0.0 & 1.500 & $-1 \cdot 7760$ & 0.4769 & 1.0730 & 0.7153 \\
\hline 0.1 & 1.500 & -1.5099 & 0.4632 & 1.0567 & 0.4795 \\
\hline 0.2 & 1.500 & $-1 \cdot 2426$ & 0.4518 & 0.9504 & 0.2724 \\
\hline 0.3 & 1.500 & -0.9842 & 0.4326 & 0.7519 & 0.0969 \\
\hline 0.4 & 1.500 & -0.7434 & 0.3968 & 0.4603 & -0.0440 \\
\hline 0.5 & 1.500 & -0.5277 & 0.3370 & 0.0761 & -0.1475 \\
\hline 0.6 & 1.500 & -0.3433 & 0.2469 & -0.3990 & -0.2106 \\
\hline 0.7 & 1.500 & -0.1953 & 0.1215 & -0.9620 & -0.2303 \\
\hline 0.8 & 1.500 & -0.0874 & -0.0429 & $-1 \cdot 6086$ & -0.2037 \\
\hline 0.9 & 1.500 & -0.0219 & -0.2486 & -2.3334 & -0.1279 \\
\hline $1 \cdot 0$ & 1.500 & -0.0000 & -0.4970 & -3.1295 & -0.0000 \\
\hline
\end{tabular}




\begin{tabular}{|c|c|c|c|c|c|}
\hline 0.0 & 1.000 & $-1 \cdot 6660$ & 0.7451 & 0.7451 & 0.7451 \\
\hline 0.1 & 1.000 & -1.4826 & 0.6871 & 0.7816 & 0.5126 \\
\hline 0.2 & 1.000 & $-1 \cdot 2658$ & 0.6514 & 0.7340 & 0.3010 \\
\hline 0.3 & 1.000 & -1.0332 & 0.6206 & 0.5950 & 0.1155 \\
\hline 0.4 & 1.000 & -0.8001 & 0.5794 & 0.3593 & -0.0384 \\
\hline 0.5 & 1.000 & -0.5799 & 0.5142 & 0.0236 & -0.1555 \\
\hline 0.6 & 1.000 & -0.3840 & 0.4138 & -0.4134 & -0.2303 \\
\hline 0.7 & 1.000 & -0.2217 & 0.2689 & -0.9509 & -0.2575 \\
\hline 0.8 & 1.000 & -0.1004 & 0.0719 & -1.5863 & -0.2318 \\
\hline 0.9 & 1.000 & -0.0254 & -0.1823 & -2.3149 & -0.1477 \\
\hline $1 \cdot 0$ & 1.000 & -0.0000 & -0.4970 & -3.1295 & -0.0000 \\
\hline 0.0 & 0.667 & $-1 \cdot 4508$ & 1.0731 & 0.4768 & 0.7153 \\
\hline 0.1 & 0.667 & -1.3771 & 0.9522 & 0.5482 & 0.5011 \\
\hline 0.2 & 0.667 & $-1 \cdot 2319$ & 0.8790 & 0.5465 & 0.2972 \\
\hline 0.3 & 0.667 & -1.0413 & 0.8272 & 0.4579 & 0.1116 \\
\hline 0.4 & 0.667 & -0.8283 & 0.7740 & 0.2717 & -0.0478 \\
\hline 0.5 & 0.667 & -0.6130 & 0.6992 & -0.0200 & -0.1727 \\
\hline 0.6 & 0.667 & -0.4126 & 0.5855 & -0.4221 & -0.2552 \\
\hline 0.7 & 0.667 & -0.2413 & 0.4189 & -0.9365 & -0.2872 \\
\hline 0.8 & 0.667 & -0.1103 & 0.1880 & $-1 \cdot 5624$ & -0.2607 \\
\hline 0.9 & 0.667 & -0.0281 & -0.1155 & -2.2957 & -0.1677 \\
\hline $1 \cdot 0$ & 0.667 & -0.0000 & -0.4970 & -3.1295 & -0.0000 \\
\hline $0 \cdot 0$ & 0.429 & $-1 \cdot 1306$ & $1 \cdot 4604$ & 0.2682 & 0.6259 \\
\hline 0.1 & 0.429 & $-1 \cdot 1939$ & 1.2582 & 0.3567 & 0.4450 \\
\hline 0.2 & 0.429 & $-1 \cdot 1414$ & 1.1340 & 0.3879 & 0.2611 \\
\hline 0.3 & 0.429 & $-1 \cdot 0089$ & 1.0520 & 0.3405 & 0.0851 \\
\hline 0.4 & 0.429 & -0.8283 & 0.9804 & 0.1974 & -0.0720 \\
\hline 0.5 & 0.429 & -0.6272 & 0.8915 & -0.0549 & -0.1991 \\
\hline 0.6 & 0.429 & -0.4293 & 0.7618 & -0.4253 & -0.2852 \\
\hline 0.7 & 0.429 & -0.2541 & 0.5716 & -0.9190 & -0.3194 \\
\hline 0.8 & 0.429 & -0.1172 & 0.3053 & -1.5369 & -0.2906 \\
\hline 0.9 & 0.429 & -0.0300 & -0.0484 & $-2 \cdot 2761$ & -0.1878 \\
\hline $1 \cdot 0$ & 0.429 & -0.0000 & -0.4970 & -3.1295 & -0.0000 \\
\hline 0.0 & 0.250 & -0.7052 & $1.90 \% 5$ & 0.1192 & 0.4769 \\
\hline 0.1 & 0.250 & -0.9330 & $1 \cdot 6050$ & 0.2066 & 0.3444 \\
\hline 0.2 & 0.250 & -0.9944 & $1 \cdot 4163$ & 0.2577 & 0.1927 \\
\hline 0.3 & 0.250 & -0.9363 & 1.2947 & 0.2424 & 0.0361 \\
\hline 0.4 & 0.250 & -0.8003 & $1 \cdot 1984$ & $0 \cdot 1359$ & -0.1111 \\
\hline 0.5 & 0.250 & -0.6227 & 1.0913 & -0.0812 & -0.2347 \\
\hline 0.6 & 0.250 & -0.4341 & 0.9426 & -0.4230 & -0.3204 \\
\hline 0.7 & 0.250 & -0.2602 & 0.7269 & -0.8982 & -0.3541 \\
\hline 0.8 & 0.250 & -0.1210 & 0.4239 & -1.5099 & -0.3214 \\
\hline $0 \cdot 9$ & 0.250 & -0.0311 & 0.0191 & $-2 \cdot 2562$ & -0.2081 \\
\hline $1 \cdot 0$ & 0.250 & -0.0000 & -0.4970 & -3.1295 & -0.0000 \\
\hline 0.0 & 0.111 & -0.1745 & $2 \cdot 4143$ & 0.0298 & 0.2682 \\
\hline 0.1 & 0.111 & -0.5946 & 1.9923 & 0.0978 & 0.1991 \\
\hline 0.2 & 0.111 & -0.7912 & $1 \cdot 7257$ & 0.1557 & 0.0919 \\
\hline 0.3 & 0.111 & -0.8236 & $1 \cdot .5551$ & 0.1633 & -0.0354 \\
\hline 0.4 & 0.111 & -0.7445 & 1.4278 & 0.0871 & -0.1651 \\
\hline 0.5 & 0.111 & -0.5995 & $1 \cdot 2984$ & -0.0993 & -0.2795 \\
\hline 0.6 & 0.111 & -0.4273 & 1.1279 & -0.4155 & -0.3608 \\
\hline 0.7 & 0.111 & -0.2597 & 0.8347 & -0.8743 & -0.3913 \\
\hline 0.8 & 0.111 & -0.1217 & 0.5437 & $-1 \cdot 4814$ & -0.3532 \\
\hline 0.9 & 0.111 & -0.0314 & 0.0869 & $-2 \cdot 2358$ & -0.2286 \\
\hline 1.0 & 0.111 & -0.0000 & -0.4970 & -3.1295 & -0.0000 \\
\hline
\end{tabular}




\section{BIBLIOGRAPHY}

(1) J.F. COUNSELL, E.B. LEES and P.J. SPENCER, "Metallurgical Chemistry", Proc. Symp. Brunel Univ., and N.P.L., 1971, Ed. O. KUBASCHEWSKI, HMSO, U.K.

(2) A.J. BRADLEY, W.F. COX a nd H.J.GOLDSCHMIDT, J. Inst. Met., 1971, $67,189$.

(3) L. ELD OFí, F. MÜLLER and O. KUBASCHEWSKI, Berich. Bunsen., 1969, 73,601 .

(4) O. KUBASCHEWSKI, private communication. 


\section{I- EXPERIMENTAL RESULTS .-}

The heats of mixing of the liquid solutions have been measured by microcalorimetry ${ }^{(1)}$ à $723 \mathrm{~K}$ for :

$$
\begin{aligned}
& 0<\mathrm{x}_{\mathrm{Zn}}<1 \quad \quad \mathrm{x}_{\mathrm{Ga}} / \mathrm{x}_{\mathrm{Sn}}=1 / 3 ; 1 / 1 ; 3 / 1 \\
& \text { and } 0<\mathrm{x}_{\mathrm{Sn}}<1 \quad ; \quad \mathrm{x}_{\mathrm{Ga}} / \mathrm{x}_{\mathrm{Zn}}=1 / 3 ; 1 / 1 ; 3 / 1
\end{aligned}
$$

The partial free energies were measured by DANILIN ${ }^{(2)}$ using an e.m.f. method in the temperature range $713-813 \mathrm{~K}$ for :

$$
0<x_{Z n}<1 ; \quad x_{G a} / x_{S n}=1 / 4 ; 1 / 1 ; 7 / 3 ; 9 / 1
$$

The phase diagram was determined by EVANS $^{(3)}$.

\section{II - COMPARISON BETWEEN EXPERIMENTAL AND CEALCULATED THERMODYNAMIC}

\section{DATA. -}

\section{a) Binary solutions :}

$$
\begin{aligned}
& \mathrm{Ga}-Z n^{(4)}\left\{\begin{aligned}
{ }_{\mathrm{H}} & =\mathrm{x}_{\mathrm{Zn}}\left(1-\mathrm{x}_{\mathrm{Zn}}\right)\left(420 \mathrm{x}_{\mathrm{Zn}}{ }^{2}+478 \mathrm{x}_{\mathrm{Zn}}+1413\right) \\
\mathrm{S}_{\mathrm{MS}}^{\mathrm{xs}} & =\mathrm{x}_{\mathrm{Zn}}\left(1-\mathrm{x}_{\mathrm{Zn}}\right)\left(-9.18456 \mathrm{x}_{\mathrm{Zn}}{ }^{3}+13.08382 \mathrm{x}_{\mathrm{Zn}}{ }^{2}\right. \\
& \left.-5.35968 \mathrm{x}_{\mathrm{Zn}}+2.49791\right)
\end{aligned}\right. \\
& S n-Z n=\left\{\begin{array}{l}
\Delta H_{M}=x_{Z n}\left(1-x_{Z n}\right)\left(2418 x_{Z n}^{2}-21 x_{Z n}+2509\right) \\
\Delta S^{x S}=x_{Z n}\left(1-x_{Z n}\right)\left(0.8317 x_{Z n}^{2}-0.12326 x_{Z n}+1.8429\right)
\end{array}\right. \\
& \mathrm{Ga}_{\mathrm{S}}(5)\left\{\begin{array}{l}
\Delta \mathrm{H}_{\mathrm{M}}=\mathrm{x}_{\mathrm{Sn}}\left(1-\mathrm{x}_{\mathrm{Sn}}\right)\left(108.318 \mathrm{x}_{\mathrm{Sn}}{ }^{2}-232.54 \mathrm{x}_{\mathrm{Sn}}+916.7\right) \\
\Delta \mathrm{S}^{\mathrm{xS}}=\mathrm{x}_{\mathrm{Sn}}\left(1-\mathrm{x}_{\mathrm{Sn}}\right) 0.25
\end{array}\right.
\end{aligned}
$$




\section{b) Ternary solutions:}

\section{1) Heats of mixing :}

The experimental values ${ }^{(1)}$ of the heats of mixing were fitted by the following equation :

$$
\begin{aligned}
& { }_{\mathrm{H}}=\left(\mathrm{x}_{\mathrm{Zn}}+\mathrm{x}_{\mathrm{Ga}}\right)^{2}\left[\mathrm{H}_{\mathrm{Ga}-\mathrm{Zn}}\right]_{\mathrm{x}_{\mathrm{Ga}}} / \mathrm{x}_{\mathrm{Zn}}+\left(\mathrm{x}_{\mathrm{Zn}}+\mathrm{x}_{\mathrm{Sn}}\right)^{2}\left[\mathrm{\Delta H}_{\mathrm{Sn}-\mathrm{Zn}}\right]_{\mathrm{x}_{\mathrm{Sn}}} / \mathrm{x}_{\mathrm{Zn}} \\
& +\left(\mathrm{x}_{\mathrm{Ga}}+\mathrm{x}_{\mathrm{Sn}}\right)\left[\mathrm{HH}_{\mathrm{Ga}-\mathrm{Sn}}{ }^{]} \mathrm{x}_{\mathrm{Ga}} / \mathrm{x}_{\mathrm{Sn}}+\mathrm{x}_{\mathrm{Ga}} \mathrm{x}_{\mathrm{Sn}} \mathrm{x}_{\mathrm{Zn}}\left[\lambda_{\mathrm{x}_{\mathrm{Zn}}}+\nu_{\mathrm{Ga}}+4 \mathrm{x}_{\mathrm{Sn}}\right]\right.
\end{aligned}
$$

which yields :

$$
\begin{aligned}
\lambda= & -4147.28 \\
\nu= & 849.46 \\
\mu= & 216.56
\end{aligned}
$$

Table 1 presents the experimental and calculated results. The differences observed are, for that case, and except for three points, with the experimental errors.

\begin{tabular}{|c|c|c|c|c|c|c|}
\hline \multirow[b]{2}{*}{${ }^{x} \mathrm{Zn}$} & \multicolumn{2}{|c|}{$1 / 3$} & \multicolumn{2}{|c|}{$1 / 1$} & \multicolumn{2}{|c|}{$3 / 1$} \\
\hline & exp & exp-calc & exp & $\exp -c a l c$ & exp & exp-calc \\
\hline 0.1 & 355 & 37 & 355 & 6 & 290 & -6 \\
\hline 0.2 & 515 & 43 & 490 & 19 & 395 & -11 \\
\hline 0.3 & 635 & 41 & 590 & 26 & 470 & -21 \\
\hline 0.4 & 710 & 29 & 655 & 21 & 535 & 1 \\
\hline 0.5 & 740 & 5 & 685 & 20 & 560 & -30 \\
\hline 0.6 & 740 & 6 & 675 & 4 & 540 & -50 \\
\hline 0.7 & 680 & -15 & 605 & -24 & 480 & -67 \\
\hline 0.8 & 560 & -27 & 475 & -50 & 380 & -61 \\
\hline 0.9 & 360 & -16 & 280 & -41 & 240 & -19 \\
\hline
\end{tabular}

\section{2) Excess, entropies of mixing :}

The excess entropies of mixing have been calculated by an equation similar to (4) with $\lambda, \nu, \mu=0$

\section{Table I}




\begin{tabular}{|c|c|c|c|c|c|c|}
\hline \multirow{2}{*}{$\int_{\mathrm{Sn}}^{\mathrm{x}} \mathrm{Ga}^{/ \mathrm{x}} \mathrm{Zn}$} & \multicolumn{2}{|c|}{$1 / 3$} & \multicolumn{2}{|r|}{$1 / 1$} & \multicolumn{2}{|c|}{$3 / 1$} \\
\hline & exp & exp-calc & $\exp$ & exp-calc & exp & exp-calc \\
\hline 0.1 & 570 & -7 & 570 & 19 & 375 & 1 \\
\hline 0.2 & 690 & 8 & 605 & 11 & 420 & 12 \\
\hline 0.3 & 730 & 10 & 610 & 12 & 425 & -6 \\
\hline 0.4 & 720 & 9 & 585 & 8 & 400 & -12 \\
\hline 0.5 & 695 & 30 & 530 & -5 & 365 & -22 \\
\hline 0.6 & 590 & 2 & 455 & -18 & 315 & -30 \\
\hline 0.7 & 475 & -9 & 360 & -29 & 255 & -31 \\
\hline 0.8 & 335 & -17 & 250 & -33 & 275 & -34 \\
\hline 0.9 & 170 & -28 & 130 & -23 & 90 & -23 \\
\hline
\end{tabular}

The calculated partial free energies of $\mathrm{Zn}$ at $723 \mathrm{~K}$ are compared to DANILIN's measurements in Table II

Table II

\begin{tabular}{|c|c|c|c|c|c|c|c|c|}
\hline$/ y_{0}$ & \multicolumn{2}{|c|}{$1 / 4$} & \multicolumn{2}{|c|}{$1 / 1$} & \multicolumn{2}{|c|}{$7 / 3$} & \multicolumn{2}{|r|}{$9 / 1$} \\
\hline & exp & exp-calc & exp & exp-calc & exp & exp-calc & exp & exp-calc \\
\hline 0.1 & -2538 & $-\quad 25$ & -2644 & 143 & -2681 & 216 & -2883 & 113 \\
\hline 0.4 & - $\quad 764$ & -110 & -953 & -44 & - 963 & +69 & -1019 & 107 \\
\hline 0.6 & $-\quad 365$ & -104 & $-\quad 476$ & $-\quad 96$ & $-\quad 582$ & -105 & - 567 & 4 \\
\hline 0.8 & -198 & $-\quad 52$ & $-\quad 245$ & $\begin{array}{l}-75 \\
-\quad 7\end{array}$ & $-\quad 264$ & $\begin{array}{l}-79 \\
-\quad 7\end{array}$ & $\begin{array}{l}-\quad 274 \\
-\end{array}$ & $\begin{array}{l}-73 \\
-\quad 7\end{array}$ \\
\hline 0.9 & $-\quad 119$ & -19 & $-\quad 124$ & -19 & $-\quad 133$ & $-\quad 28$ & $-\quad 137$ & $-\quad 33$ \\
\hline
\end{tabular}

UI - COMPARISON BETWEEN THE EXPERIMENTAL AND CALCULATED PHASE DIAGRAM.The Ga-Sn, Ga-Zn and Zn-Sn phases diagrams are simple eutectictype with no appreciable solid solution except in the $\mathrm{Sn}$ rich corner in the $\mathrm{Sn}-\mathrm{Zn}$ system. The liquidus points of the ternary system are compared to the experimental results ${ }^{(3)}$ in Table III. The aqreement is very satisfactory except in the Ga rich corner. A calculated diagram is shown in figure 1 . 
TABLE III

\begin{tabular}{|c|c|c|c|c|}
\hline$x_{G a}$ & ${ }^{x_{S n}}$ & $\mathrm{x}_{\mathrm{Zn}}$ & exp & exp-calc \\
\hline \multicolumn{5}{|c|}{$\mathrm{liq} \rightleftarrows \mathrm{Zn}$} \\
\hline 0.833 & 0.067 & 0.100 & 357 & -1 \\
\hline 0.819 & 0.045 & 0.136 & 383 & 2 \\
\hline 0.804 & 0.024 & 0.172 & 396 & -4 \\
\hline 0.670 & 0.181 & 0.149 & 398 & 1 \\
\hline 0.662 & 0.162 & 0.176 & 414 & 3 \\
\hline 0.283 & 0.597 & 0.120 & 420 & -4 \\
\hline 0.493 & 0.343 & 0.164 & 421 & 9 \\
\hline 0.575 & 0.229 & 0.196 & 430 & 8 \\
\hline 0.486 & 0.321 & 0.194 & 436 & 10 \\
\hline 0.279 & 0.572 & 0.149 & 438 & 11 \\
\hline 0.556 & 0.183 & 0.261 & 455 & 6 \\
\hline 0.270 & 0.515 & 0.215 & 472 & 14 \\
\hline 0.539 & 0.139 & 0.322 & 476 & 7 \\
\hline 0.261 & 0.460 & 0.279 & 498 & 15 \\
\hline \multicolumn{5}{|c|}{$\mathrm{liq} \rightleftharpoons \mathrm{Ga}$} \\
\hline 0.873 & 0.045 & 0.082 & 286 & 1 \\
\hline 0.867 & 0.047 & 0.086 & 292.5 & 21.5 \\
\hline 0.911 & 0.024 & 0.065 & 295 & 22 \\
\hline 0.916 & 0.030 & 0.054 & 296 & 9 \\
\hline 0.956 & 0.012 & 0.032 & 297 & 14 \\
\hline 0.924 & 0.012 & 0.064 & 300 & 29 \\
\hline 0.954 & 0.024 & 0.022 & 300 & 13 \\
\hline \multicolumn{5}{|c|}{$\mathrm{liq} \rightleftarrows \mathrm{Sn}$} \\
\hline 0.845 & 0.087 & 0.068 & 299 & 4 \\
\hline 0.859 & 0.107 & 0.034 & 305 & 0 \\
\hline 0.691 & 0.223 & 0.086 & 350 & 9 \\
\hline 0.710 & 0.264 & 0.026 & 357 & 1 \\
\hline 0.528 & 0.458 & 0.014 & 360 & 2 \\
\hline 0.509 & 0.396 & 0.095 & 386 & 0 \\
\hline 0.516 & 0.416 & 0.068 & 388 & -1 \\
\hline 0.522 & 0.436 & 0.042 & 390 & -2 \\
\hline 0.528 & 0.458 & 0.014 & 395 & 1 \\
\hline 0.286 & 0.622 & 0.092 & 422 & -5 \\
\hline 0.292 & 0.611 & 0.047 & 430 & -2 \\
\hline 0.297 & 0.687 & 0.016 & 436 & 0 \\
\hline
\end{tabular}




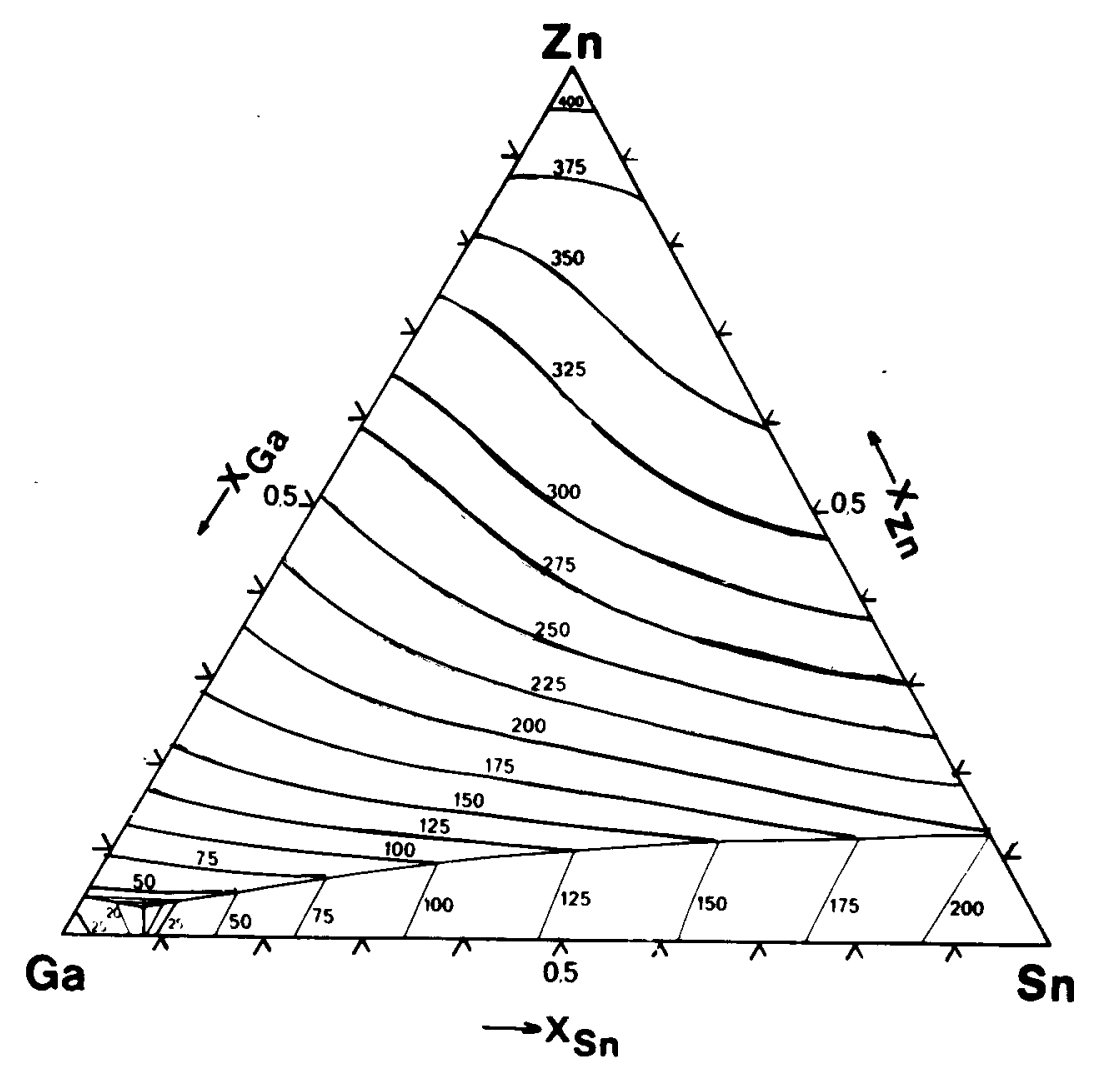




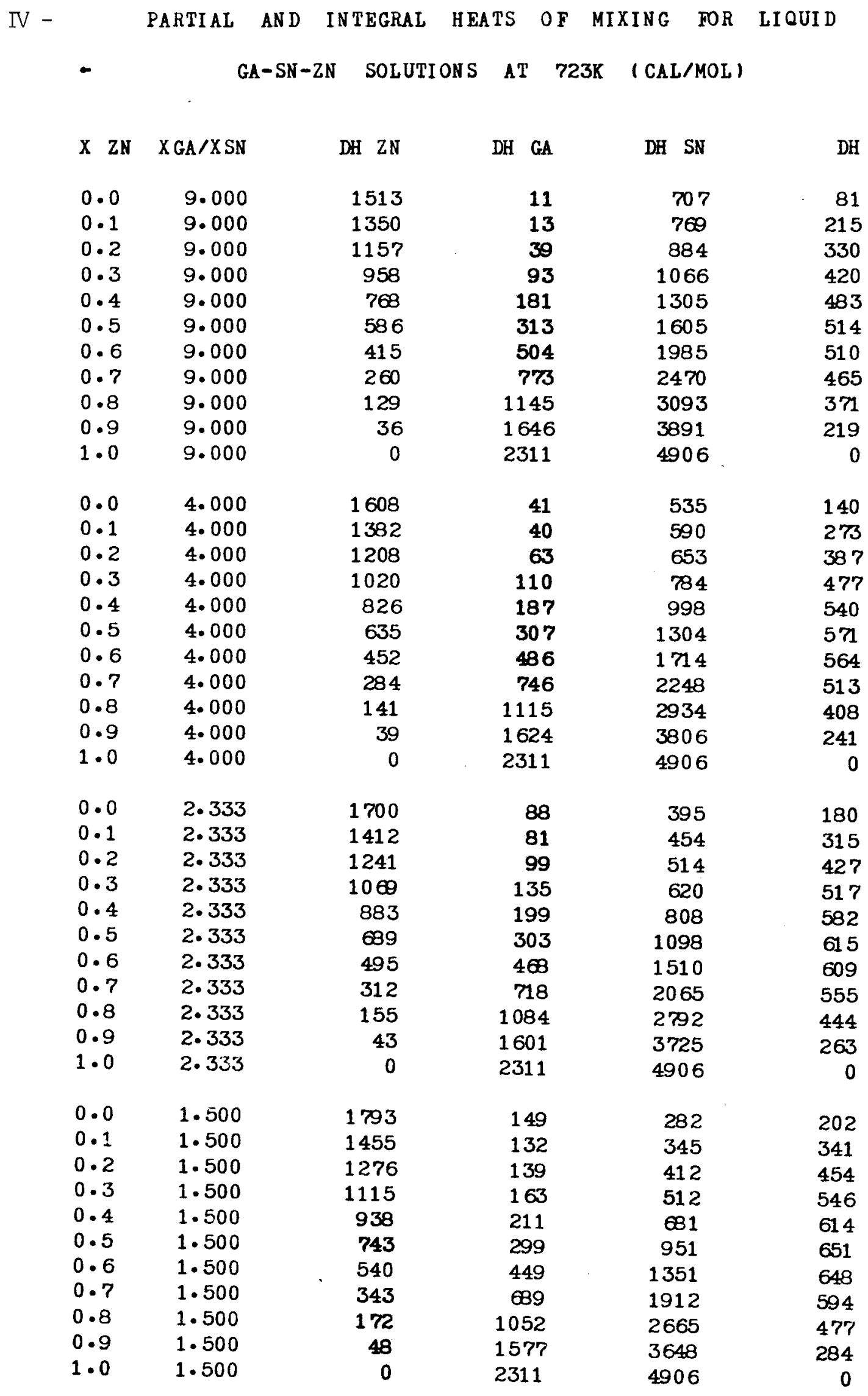




\begin{tabular}{|c|c|c|c|c|c|}
\hline 0.0 & 1.000 & 1887 & 222 & 191 & 207 \\
\hline 0.1 & 1.000 & 1517 & 193 & 255 & 353 \\
\hline 0.2 & 1.000 & 1323 & 184 & 332 & 471 \\
\hline 0.3 & 1.000 & 1164 & 190 & 432 & 567 \\
\hline 0.4 & 1.000 & 992 & 220 & 589 & 639 \\
\hline 0.5 & 1.000 & 797 & 291 & 841 & 681 \\
\hline 0.6 & 1.000 & 586 & 426 & 1225 & 682 \\
\hline 0.7 & 1.000 & 376 & 657 & 1782 & 629 \\
\hline 0.8 & 1.000 & 189 & 1018 & 2550 & 508 \\
\hline 0.9 & 1.000 & 53 & 1553 & 3576 & 304 \\
\hline $1 \cdot 0$ & 1.000 & 0 & 2311 & 4906 & 0 \\
\hline 0.0 & 0.667 & 1987 & 309 & 121 & 196 \\
\hline $0 \cdot 1$ & 0.667 & 1599 & 260 & 183 & 353 \\
\hline 0.2 & 0.667 & 1386 & 230 & 265 & 478 \\
\hline 0.3 & 0.667 & 1220 & 215 & 367 & 581 \\
\hline 0.4 & 0.667 & 1047 & 226 & 517 & 659 \\
\hline 0.5 & 0.667 & 850 & 280 & 753 & 207 \\
\hline 0.6 & 0.667 & 631 & 401 & 1121 & 72 \\
\hline 0.7 & 0.667 & 409 & 623 & 1668 & 661 \\
\hline 0.8 & 0.667 & 208 & 983 & 2446 & 538 \\
\hline 0.9 & 0.667 & 59 & 1528 & 3506 & 324 \\
\hline 1.0 & 0.667 & 0 & 2311 & 4906 & 0 \\
\hline 0.0 & $4 \cdot 286$ & 1598 & 37 & 552 & 134 \\
\hline 0.1 & 4.286 & 1379 & 37 & 607 & $2 ఱ B$ \\
\hline $0 \cdot 2$ & 4.286 & 1204 & 60 & 672 & 381 \\
\hline 0.3 & 4.286 & 1014 & 107 & 807 & 472 \\
\hline 0.4 & 4.286 & 820 & 186 & 1024 & 535 \\
\hline 0.5 & 4.286 & 630 & 307 & 1331 & 565 \\
\hline 0.6 & 4.286 & 448 & 488 & 1740 & 559 \\
\hline 0.7 & $4 \cdot 286$ & 281 & 749 & 2270 & 508 \\
\hline 0.8 & 4.286 & 139 & 1118 & 2950 & 404 \\
\hline 0.9 & 4.286 & 39 & 1626 & 3815 & 239 \\
\hline $1 \cdot 0$ & $4 \cdot 286$ & 0 & 2311 & 4906 & 0 \\
\hline 0.0 & 2.500 & 1687 & 80 & 413 & 175 \\
\hline 0.1 & 2.500 & 1407 & 74 & 472 & 310 \\
\hline 0.2 & 2.500 & 1236 & 93 & 531 & 422 \\
\hline 0.3 & 2.500 & 1062 & 132 & 639 & 512 \\
\hline 0.4 & 2.500 & 875 & 197 & 830 & 577 \\
\hline 0.5 & 2.500 & 681 & 304 & 1123 & 610 \\
\hline 0.6 & 2.500 & 489 & 47 & 1536 & 603 \\
\hline 0.7 & 2.500 & 308 & 722 & 2089 & 549 \\
\hline 0.8 & 2.500 & 153 & 1088 & 2811 & 439 \\
\hline 0.9 & 2.500 & 43 & 1604 & 3736 & 260 \\
\hline $1 \cdot 0$ & 2.500 & 0 & 2311 & 4906 & 0 \\
\hline 0.0 & 0.111 & 2353 & 647 & 8 & 72 \\
\hline 0.1 & 0.111 & 1958 & 519 & 44 & 278 \\
\hline 0.2 & 0.111 & 167 & 414 & 111 & 447 \\
\hline 0.3 & 0.111 & 1446 & 328 & 204 & 585 \\
\hline 0.4 & 0.111 & 1236 & 270 & 337 & 693 \\
\hline 0.5 & 0.111 & 1013 & 260 & 543 & 764 \\
\hline 0.6 & 0.111 & 767 & 329 & 872 & 788 \\
\hline 0.7 & 0.111 & 509 & 517 & 1390 & 747 \\
\hline $\begin{array}{l}0.8 \\
0.9\end{array}$ & $\begin{array}{l}0.111 \\
0.111\end{array}$ & $\begin{array}{r}265 \\
77\end{array}$ & $\begin{array}{r}873 \\
1451\end{array}$ & $\begin{array}{l}2174 \\
3313\end{array}$ & $\begin{array}{l}621 \\
382\end{array}$ \\
\hline $1 \cdot 0$ & 0.111 & 0 & 2311 & 4906 & 0 \\
\hline
\end{tabular}


PARTIAL AND INTEGRAL EXCESS ENTROPIES OF MIXING FOR

\section{LIQUID GA-SN-ZN SOLUTIONS AT 723K (CAL/MOL)/K}

\begin{tabular}{|c|c|c|c|c|c|}
\hline$X \quad Z N$ & X GA $/ X$ SN & SXS ZN & SXS GA & SX S SN & SXS \\
\hline 0.0 & 9.000 & 2.4099 & 0.0025 & 0.2025 & 0.0225 \\
\hline 0.1 & 9.000 & $1 \cdot 4361$ & 0.0528 & 0.1819 & 0.2027 \\
\hline 0.2 & 9.000 & 1.0927 & 0.1078 & 0.2557 & 0.3166 \\
\hline 0.3 & 9.000 & 0.9474 & 0.1466 & 0.3845 & 0.4035 \\
\hline 0.4 & 9.000 & 0.7985 & $0 \cdot 2190$ & 0.5502 & 0.4706 \\
\hline 0.5 & 9.000 & 0.5896 & 0.3888 & 0.7566 & 0.5076 \\
\hline 0.6 & 9.000 & 0.3491 & 0.6892 & 1.0163 & 0.498 \\
\hline 0.7 & 9.000 & 0.1387 & 1.0872 & 1.3387 & 0.430 \\
\hline 0.8 & 9.000 & 0.0126 & 1.4574 & $1 \cdot 7211$ & 0.3068 \\
\hline 0.9 & 9.000 & -0.0160 & $1 \cdot 5621$ & $2 \cdot 1411$ & 0.1476 \\
\hline 1.0 & 9.000 & -0.0000 & 1.0375 & $2 \cdot 5514$ & 0.0000 \\
\hline 0.0 & 4.000 & 2.3269 & 0.0100 & 0.1600 & 0.0400 \\
\hline 0.1 & 4.000 & 1.4073 & 0.0625 & 0.1474 & 0.2123 \\
\hline 0.2 & 4.000 & 1.1276 & 0.1069 & 0.2006 & 0.3261 \\
\hline 0.3 & 4.000 & 0.9918 & 0.1386 & 0.3006 & 0.4172 \\
\hline 0.4 & 4.000 & 0.8196 & 0.2203 & 0.4468 & 0.487 \\
\hline 0.5 & 4.000 & 0.5867 & 0.4110 & 0.6486 & 0.522 \\
\hline 0.6 & 4.000 & 0.3385 & 0.7251 & 0.9168 & 0.5085 \\
\hline 0.7 & 4.000 & 0.1356 & 1.1103 & $1 \cdot 2560$ & 0.4367 \\
\hline 0.8 & 4.000 & 0.0188 & $1 \cdot 4389$ & $1 \cdot 6593$ & 0.3117 \\
\hline 0.9 & 4.000 & -0.0101 & 1.5067 & 2.1042 & 0.1535 \\
\hline $1 \cdot 0$ & 4.000 & -0.0000 & 1.0375 & $2 \cdot 5514$ & 0.0000 \\
\hline 0.0 & 2.333 & $2 \cdot 2489$ & 0.0225 & 0.1225 & 0.0525 \\
\hline 0.1 & 2.333 & $1 \cdot 3845$ & 0.0750 & 0.1200 & 0.2181 \\
\hline 0.2 & 2.333 & $1 \cdot 1568$ & 0.1079 & 0.1682 & 0.3321 \\
\hline 0.3 & 2.333 & 1.0234 & 0.1369 & 0.2510 & 0.4268 \\
\hline 0.4 & 2.333 & 0.8287 & 0.2342 & 0.3804 & 0.4983 \\
\hline 0.5 & 2.333 & 0.5785 & 0.4469 & 0.5733 & 0.5317 \\
\hline 0.6 & $2 \cdot 333$ & 0.3297 & $0.76 \% 7$ & 0.8417 & 0.5138 \\
\hline 0.7 & 2.333 & 0.1368 & 1.1290 & $1 \cdot 1880$ & 0.4398 \\
\hline 0.8 & $2 \cdot 333$ & 0.0278 & $1 \cdot 4094$ & 1.6037 & 0.3158 \\
\hline 0.9 & 2.33 .3 & -0.0040 & $1 \cdot 4444$ & 2.0684 & 0.1596 \\
\hline $1 \cdot 0$ & 2.333 & -0.0000 & 1.0375 & $2 \cdot 5514$ & 0.0000 \\
\hline 0.0 & 1.500 & 2.1759 & 0.0400 & 0.0900 & 0.0600 \\
\hline 0.1 & 1.500 & 1.3740 & 0.0897 & 0.0961 & 0.2204 \\
\hline 0.2 & 1.500 & $1 \cdot 1860$ & 0.1100 & 0.1434 & 0.3359 \\
\hline 0.3 & 1.500 & 1.0424 & 0.1427 & $0.21 \oplus$ & 0.4334 \\
\hline 0.4 & 1.500 & 0.8249 & 0.2622 & 0.3355 & 0.5049 \\
\hline 0.5 & 1.500 & 0.5652 & 0.4957 & 0.5205 & 0.5354 \\
\hline 0.6 & 1.500 & 0.3231 & 0.8130 & 0.7851 & 0.5146 \\
\hline 0.7 & 1.500 & 0.1425 & $1 \cdot 1383$ & $1 \cdot 1318$ & 0.4405 \\
\hline 0.8 & 1.500 & 0.0393 & 1.3658 & $1 \cdot 5532$ & 0.3196 \\
\hline 0.9 & 1.500 & 0.0024 & 1.3744 & 2.0337 & 0.1660 \\
\hline $1 \cdot 0$ & 1.500 & -0.0000 & 1.0375 & 2.5514 & 0.0000 \\
\hline
\end{tabular}




\begin{tabular}{|c|c|c|c|c|c|}
\hline 0.0 & 1.000 & 2.1079 & 0.0625 & 0.0625 & 0.0625 \\
\hline 0.1 & 1.000 & 1.3783 & 0.1058 & 0.0753 & 0.2193 \\
\hline 0.2 & 1.000 & 1.2153 & 0.1141 & 0.1221 & 0.3375 \\
\hline 0.3 & 1.000 & 1.0477 & 0.1596 & 0.1913 & 0.4371 \\
\hline 0.4 & 1.000 & 0.8089 & 0.3071 & 0.3045 & 0.5070 \\
\hline 0.5 & 1.000 & 0.5488 & 0.5554 & 0.4839 & 0.5342 \\
\hline 0.6 & 1.000 & 0.3204 & 0.8548 & 0.7427 & 0.5118 \\
\hline 0.7 & 1.000 & 0.1531 & 1.1313 & 1.0849 & 0.4396 \\
\hline 0.8 & 1.000 & 0.0529 & 1.3042 & 1.5069 & 0.3234 \\
\hline 0.9 & 1.000 & 0.0089 & 1.2959 & 1.9999 & 0.1728 \\
\hline 1.0 & 1.000 & 0.0000 & 1.0375 & 2.5514 & 0.0000 \\
\hline 0.0 & 0.667 & 2.0449 & 0.0900 & 0.0400 & 0.0600 \\
\hline 0.1 & 0.667 & $1 \cdot 3993$ & 0.1222 & 0.0576 & 0.2150 \\
\hline 0.2 & 0.667 & 1.2405 & 0.1230 & 0.1034 & 0.3371 \\
\hline 0.3 & 0.667 & 1.0373 & 0.1938 & 0.1717 & 0.4376 \\
\hline 0.4 & 0.667 & 0.7832 & 0.3705 & 0.2839 & 0.5044 \\
\hline 0.5 & 0.667 & 0.5332 & 0.6197 & 0.4596 & 0.5284 \\
\hline 0.6 & 0.667 & 0.3237 & 0.8821 & 0.7111 & 0.5060 \\
\hline 0.7 & 0.667 & 0.1687 & 1.0986 & 1.0451 & 0.4380 \\
\hline 0.8 & 0.667 & 0.0683 & 1.2197 & 1.4638 & 0.3278 \\
\hline 0.9 & 0.667 & 0.0153 & 1.2080 & 1.9667 & 0.1801 \\
\hline 1.0 & 0.667 & 0.0000 & 1.0375 & 2.5514 & 0.0000 \\
\hline 0.0 & 4.286 & 2.3356 & 0.0089 & 0.1644 & 0.0383 \\
\hline 0.1 & 4.286 & 1.4103 & 0.0613 & 0.1506 & 0.2114 \\
\hline 0.2 & 4.286 & 1.1243 & 0.1069 & 0.2050 & 0.3252 \\
\hline 0.3 & 4.286 & 0.9876 & 0.1391 & 0.3075 & 0.4160 \\
\hline 0.4 & 4.286 & 0.8179 & 0.2195 & 0.4558 & 0.4857 \\
\hline 0.5 & 4.286 & 0.5873 & 0.4080 & 0.6584 & 0.5213 \\
\hline 0.6 & 4.286 & 0.3396 & 0.7209 & 0.9262 & 0.5076 \\
\hline 0.7 & 4.286 & 0.1357 & 1.1080 & 1.2642 & 0.4362 \\
\hline 0.8 & 4.286 & 0.0180 & 1.4414 & 1.6656 & 0.3112 \\
\hline 0.9 & 4.286 & -0.0108 & 1.5130 & $2 \cdot 1081$ & 0.1528 \\
\hline 1.0 & 4.286 & $\because 0.0000$ & 1.0375 & 2.5514 & 0.0000 \\
\hline 0.0 & 2.500 & 2.2597 & 0.0204 & 0.1276 & 0.0510 \\
\hline 0.1 & 2.500 & 1.3871 & 0.0731 & 0.1237 & 0.2175 \\
\hline 0.2 & 2.500 & 1.1527 & 0.1077 & 0.1722 & 0.3314 \\
\hline 0.3 & 2.500 & 1.0196 & 0.1368 & 0.2569 & 0.4256 \\
\hline 0.4 & 2.500 & 0.8282 & 0.2314 & 0.3883 & 0.4970 \\
\hline 0.5 & 2.500 & 0.5800 & 0.4410 & 0.5825 & 0.5307 \\
\hline 0.6 & 2.500 & 0.3308 & 0.7613 & 0.8511 & 0.5133 \\
\hline 0.7 & 2.500 & 0.1363 & 1.1263 & 1.1969 & 0.4395 \\
\hline 0.8 & 2.500 & 0.0264 & 1.4144 & 1.6113 & 0.3152 \\
\hline 0.9 & 2.500 & -0.0049 & $1 \cdot 4537$ & 2.0735 & 0.1587 \\
\hline 1.0 & 2.500 & -0.0000 & 1.0375 & 2.5514 & 0.0000 \\
\hline 0.0 & 0.111 & 1.8859 & 0.2025 & 0.0025 & 0.0225 \\
\hline 0.1 & 0.111 & 1.5039 & 0.1944 & 0.0210 & 0.1849 \\
\hline 0.2 & 0.111 & 1.1995 & 0.2957 & 0.0694 & 0.3135 \\
\hline 0.3 & 0.111 & 0.9460 & 0.4116 & 0.1501 & 0.4072 \\
\hline 0.4 & 0.111 & 0.7355 & 0.4997 & 0.2663 & 0.4680 \\
\hline 0.5 & 0.111 & 0.5512 & 0.5650 & 0.4270 & 0.4960 \\
\hline 0.6 & 0.111 & 0.3854 & 0.6213 & 0.6467 & 0.4889 \\
\hline 0.7 & 0.111 & 0.2385 & $0 . \oplus 332$ & 0.9445 & 0.4425 \\
\hline 0.8 & 0.111 & 0.1170 & 0.7644 & $1 \cdot 3434$ & 0.3507 \\
\hline 0.9 & 0.111 & 0.0323 & 0.8782 & 1.8695 & 0.2061 \\
\hline 1.0 & 0.111 & 0.0000 & 1.0375 & 2.5514 & 0.0000 \\
\hline
\end{tabular}


(1) M. GAMBino, J.P. BROS and I. ANSARA, Rev. Int. Htes Temp. et Réfract., $1973,10,1,5$.

(2) N.V. DANILIN, S.P. YATSENKO, V.J. KONONENKO and BRUZHJINA, "Propriétés du Gallium en solution et sous forme d'alliages", p. 83, Inst. Khim. Akad. Nauk SSSR, Ural. Fil., USSR.

(3) R. EVANS and R. JOFFEE, Trans. Met. Soc. AIME, 1952, 296, 163.

(4) J.P. BROS and M. LAFFITTE, J. Chim. Phys., 1970, 67, 1636.

(5) M. GAMBINO, J.P. BROS and I. ANSARA, Colloque International du CNRS "Thermochimie", Marseille, 1971, 201.

(6) R. HULTGREN, P.D. DESRE, D.T. HAWKINS, M. GLEISER and K.K. KELty, "Selected Values of the Thermodynamic Properties of Binary Alloys", 1973, Amer. Soc. Metals, Metals Park, Ohio, USA. 
Imprimeur : Ecole Nationale Supérieure d'Electrochimie et d'Electrométallurgie Domaine Universitaire

38 - SAINT MARTIN D'HERES 